\title{
Multiresolution Monogenic Signal Analysis Using the Riesz-Laplace Wavelet Transform
}

\author{
Michael Unser, Fellow, IEEE, Daniel Sage, and Dimitri Van De Ville, Member, IEEE
}

\begin{abstract}
The monogenic signal is the natural 2-D counterpart of the 1-D analytic signal. We propose to transpose the concept to the wavelet domain by considering a complexified version of the Riesz transform which has the remarkable property of mapping a real-valued (primary) wavelet basis of $L_{2}\left(\mathbb{R}^{2}\right)$ into a complex one. The Riesz operator is also steerable in the sense that it give access to the Hilbert transform of the signal along any orientation. Having set those foundations, we specify a primary polyharmonic spline wavelet basis of $L_{2}\left(\mathbb{R}^{2}\right)$ that involves a single Mexican-hat-like mother wavelet (Laplacian of a B-spline). The important point is that our primary wavelets are quasi-isotropic: they behave like multiscale versions of the fractional Laplace operator from which they are derived, which ensures steerability. We propose to pair these real-valued basis functions with their complex Riesz counterparts to specify a multiresolution monogenic signal analysis. This yields a representation where each wavelet index is associated with a local orientation, an amplitude and a phase. We give a corresponding wavelet-domain method for estimating the underlying instantaneous frequency. We also provide a mechanism for improving the shift and rotation-invariance of the wavelet decomposition and show how to implement the transform efficiently using perfect-reconstruction filterbanks. We illustrate the specific feature-extraction capabilities of the representation and present novel examples of wavelet-domain processing; in particular, a robust, tensor-based analysis of directional image patterns, the demodulation of interferograms, and the reconstruction of digital holograms.
\end{abstract}

Index Terms-Analytic signal, directional image analysis, Hilbert transform, monogenic signal, polyharmonic splines, Riesz transform, steerable filters, wavelet transform.

\section{INTRODUCTION}

$\mathbf{T}$ HE analytic signal is a complex extension of a 1-D signal that is based upon the Hilbert transform; it was introduced to signal theory by Gabor in 1946 [1]. This representation gives access to the instantaneous amplitude and phase of a signal and is widely used in applications involving some kind of amplitude or frequency modulations [2], [3]. This type of AM/FM analysis can also be performed in a multiresolution framework using Kingsbury's dual-tree wavelet transform, which consists

Manuscript received October 03, 2008; revised May 27, 2009. First published July 14, 2009; current version published October 16, 2009. This work was supported in part by the Swiss National Science Foundation (MU, KB) under Grant 200020-109415 and in part by the Center for Biomedical Imaging (DVDV) of the Geneva-Lausanne Universities and the EPFL, as well as the foundations Leenaards and Louis-Jeantet. The associate editor coordinating the review of this manuscript and approving it for publication was Prof. Minh N. Do.

The authors are with the Biomedical Imaging Group (BIG), École Polytechnique Fédérale de Lausanne (EPFL), CH-1015 Lausanne, Switzerland (e-mail: michael.unser@epfl.ch; daniel.sage@epfl.ch; dimitri.vandeville@epfl.ch).

Color versions of one or more of the figures in this paper are available online at http://ieeexplore.ieee.org.

Digital Object Identifier 10.1109/TIP.2009.2027628 of two wavelet transforms which are (approximately) Hilbert transforms of one another [4]-[7]. An exact implementation is actually possible within the framework of fractional splines [8], which is closed under fractional differentiation. ${ }^{1}$

Several attempts to generalize the analytic signal to two dimensions have been reported in the literature [9]-[11]. The first class of representations relies on a separable application of the Hilbert transform along the $x$ and $y$ coordinates. In the approach of Hahn [9], the analytic signal is represented by two complex components whose Fourier spectrum is restricted to the upper-right and lower-right quadrant, respectively. The transposition of this approach to the wavelet domain results in a 2-D dual-tree complex wavelet transform that has a redundancy of four. The transform allows for a separation into six distinct orientation channels via a proper combination of positive and negative frequency bands [4], [12]. The clear advantage of this type of representation over the traditional real-valued separable wavelet transforms is improved shift-invariance (thanks to the notions of wavelet-domain amplitude and phase) and better orientation selectivity [4], [5], [12]. There is also an alternative of the 2-D analytic signal—due to Bülow and Sommer [10]—-that starts with the same separable Hilbert-transform constituents, but combines them into four "quadrature" components that are manipulated using a more sophisticated quaternion algebra. The transfer of this concept to the wavelet domain leads the dual-tree quaternion wavelet transform [13], which is well suited for the coherent processing of relative positional information.

The third generalization of the analytic signal-called the monogenic signal — was introduced by Felsberg and Sommer in 2001 [11]. Its distinguishing property is that the underlying feature extraction process (phase, amplitude and orientation estimation) is truly rotation-invariant which explains its success for image analysis. The method is build around the Riesz transform which is the vector-valued extension of the Hilbert transform favored by mathematicans [14]. To make an analogy, the Riesz transform is to the Hilbert transform what the gradient is to the derivative operator. The image-processing applications of the monogenic signal are numerous: they include contour detection and local structure analysis [15], [16]; stereo, motion estimation, and image registration [17], [18]; as well as image segmentation and phase-contrast imaging [19]. The monogenic signal is also closely linked to the spiral quadrature phase transform in optics which constitutes a powerful tool for the processing of fringe patterns and the demodulation of interferograms [20]-[22]. Recently, Metikas and Olhede have transposed the approach to the

\footnotetext{
${ }^{1}$ The Hilbert transform can be interpreted as the anti-symmetric (fractional) derivative of order 0 , while the identity is the symmetric derivative of order 0 . Likewise, we may view the Riesz transform, which is central to this work, as the (fractional) gradient of order 0 .
} 
wavelet-domain, albeit within the framework of the continuous wavelet transform which is an overly-redundant representation [23]. In practice, the monogenic analysis is often performed on some bandpass-filtered versions of the input signal which also leads to the idea of multiresolution [15], [24]. Our goal in this paper is to fill the gap by presenting the minimally-redundant wavelet counterpart of Felsberg's monogenic signal representation. The nontrivial aspect here is that the multiscale versions of the signal are critically sampled and that the scheme is fully reversible (exact reconstruction property). ${ }^{2}$

The main features of the proposed "Riesz-Laplace" wavelet decomposition are as follows.

- The wavelet transform has three monogenic components for any scale/location index.

- The primary mother analysis wavelet is Laplacian-like and essentially isotropic, while the two others are the components of the Riesz transform of the former. In our formulation, the two Riesz wavelets are combined into a single complex transform.

- The three wavelet components give access to the local orientation - that is, the dominant orientation of image features in a local neighborhood-as well as to the key $\mathrm{AM} / \mathrm{FM}$ parameters in the preferred orientation: amplitude, phase, and instantaneous frequency. These latter parameters are specific to the monogenic formalism and are not accessible in a conventional wavelet transform.

- The monogenic wavelet analysis is essentially rotation-invariant because of: 1) the isotropy of the mother wavelet, and 2) the fact that the Riesz transform is steerable.

- The wavelet transform has a fast filterbank algorithm. In fact, the decomposition involves the concatenation of two wavelet bases of $L_{2}\left(\mathbb{R}^{2}\right)$, the second of which is complexvalued.

The paper is organized as follows. In Section II, we review the key properties of the complex Riesz transform, and reinterpret Felsberg's monogenic signal using the directional Hilbert transform; we also present a more robust, tensor-based estimation of the local orientation. In Section III, we use the invariance properties of the complex Riesz operator to construct a new complex wavelet basis of $L_{2}\left(\mathbb{R}^{2}\right)$ through the unitary mapping of a primary real-valued wavelet transform. Our primary wavelet basis involves a single Mexican-hat-like mother wavelet. It is specified within a polyharmonic spline framework that forces the wavelet to replicate the isotropic behavior of the (fractional) Laplacian. This ensures that the secondary complex wavelet, which is the Riesz transform of the former, is steerable. In Section IV, we propose to use the so-defined pair of Riesz-Laplace wavelet transforms to specify a multiresolution monogenic signal analysis. We also show how to extract wavelet-domain information that characterizes the local structure of the image (orientation, coherency), as well as its modulation/localization properties (amplitude, phase and spatial frequency). In Section V, we propose two simple solutions for improving the shift and rotation-invariance of the decomposition.

${ }^{2}$ While this paper was under review, the authors became aware of the work of Held et al. that introduces a similar construction based on isotropic wavelet frames in $L_{2}\left(\mathbb{R}^{n}\right)$ [25]; in the process, they also provide a hypercomplex generalization of the monogenic signal for dimensions higher than two.
We illustrate our method with concrete examples of wavelet-domain monogenic signal analyses, including a novel application of wavelets to coherent optical imaging.

\section{RiesZ TRANSFORM AND THE MONOGENIC SignAL}

We start with a brief review of the Hilbert transform and the analytic signal formalism and then proceed with the extension of those concepts to two dimensions. In particular, we present an alternative formulation of Feldberg's monogenic signal representation that rests upon a "complexified" version of the Riesz transform which is due to Larkin [21]. This operator has a number of remarkable of properties that will be exploited in our construction.

\section{A. Hilbert Transform}

The 1-D Hilbert transform is the linear, shift-invariant operator $\mathcal{H}$ that maps all 1-D cosine functions into their corresponding sine functions. It is an allpass filter that is characterized by the transfer function $H(\omega)=-j \operatorname{sign}(\omega)=-j \omega /|\omega|$. Its impulse response (in the sense of distributions) is $h(x)=$ $1 /(\pi x)$. Note that $h(x)$ is neither in $L_{1}(\mathbb{R})$ nor in $L_{2}(\mathbb{R})$ due to the singularity at the origin. The other important mathematical feature is the slow decay of $h(x)$ which implies that the Hilbert transform is a nonlocal operator; in particular, it will generally map compactly supported functions into noncompactly supported ones.

The Hilbert transform plays a central role in the analytic signal formalism which is a powerful tool for AM/FM analysis. Given a real valued 1-D signal $f(x)$, one defines the complex analytic signal

$$
f_{\text {anal }}(x)=f(x)+j \mathcal{H} f(x)=A(x) e^{j \xi(x)} .
$$

The advantage of this representation is that it allows one to retrieve the time-varying amplitude and instantaneous frequency of an AM/FM signal of the form $f(x)=A(x) \cos \left(2 \pi \nu(x) x+\xi_{0}\right)$, where $A(x)$ and $\nu(x)$ are slowly-varying. Specifically, $A(x)=\left|f_{\text {anal }}(x)\right|$ and $\nu(x)=\mathrm{d} \xi(x) / \mathrm{d} x$.

\section{B. Riesz Transform}

The Riesz transform is the natural multidimensional extension of the Hilbert transform [14]. It is the scalar-to-vector signal transformation whose frequency response is $-j \omega /\|\omega\|$. In 2-D, which is the case of interest here, it can be expressed as

$$
\mathbf{f}_{\mathrm{R}}(\boldsymbol{x})=\left(\begin{array}{c}
f_{1}(\boldsymbol{x}) \\
f_{2}(\boldsymbol{x})
\end{array}\right)=\left(\begin{array}{l}
h_{x} * f(\boldsymbol{x}) \\
h_{y} * f(\boldsymbol{x})
\end{array}\right)
$$

where $f(\boldsymbol{x})$ with $\boldsymbol{x}=(x, y)$ is the input signal, and where the filters $h_{x}$ and $h_{y}$ are characterized by the 2-D frequency responses $H_{x}(\boldsymbol{\omega})=-j \omega_{x} /\|\boldsymbol{\omega}\|$ and $H_{y}(\boldsymbol{\omega})=-j \omega_{y} /\|\boldsymbol{\omega}\|$ with $\boldsymbol{\omega}=\left(\omega_{x}, \omega_{y}\right)$. By using the well-known property that $\mathcal{F}\{1 /\|\boldsymbol{x}\|\}(\boldsymbol{\omega})=2 \pi /\|\boldsymbol{\omega}\|$ and performing a partial differentiation with respect to $x$ or $y$, we readily derive the corresponding impulse responses $h_{x}(x)=x /\left(2 \pi\|x\|^{3}\right)$ and $h_{x}(x)=y /\left(2 \pi\|x\|^{3}\right)$.

For the present work, we have chosen to work with a "complexified" version of the Riesz transform that combines the two 
Riesz components into a single complex signal; it was introduced by Larkin in optics under the name of the spiral phase quadrature transform [20], [21]. The corresponding definition of the 2-D complex Riesz operator is

$$
\mathcal{R} f(\boldsymbol{x}) \stackrel{\mathcal{F}}{\longleftrightarrow} \frac{\left(-j \omega_{x}+\omega_{y}\right)}{\|\boldsymbol{\omega}\|} \hat{f}(\boldsymbol{\omega})
$$

where $\hat{f}(\boldsymbol{\omega})=\int_{\mathbb{R}^{2}} f(\boldsymbol{x}) e^{-j\langle\boldsymbol{\omega}, \boldsymbol{x}\rangle} \mathrm{d} x \mathrm{~d} y$ is the 2-D Fourier transform of $f$. The equivalent space-domain formulation is

$$
\begin{aligned}
\mathcal{R} f(\boldsymbol{x}) & =\left(\left(h_{x}+j h_{y}\right) * f\right)(\boldsymbol{x}) \\
& =\frac{1}{2 \pi} \int_{\mathbb{R}^{2}} \frac{x^{\prime}+j y^{\prime}}{\left\|\boldsymbol{x}^{\prime}\right\|^{3}} f\left(\boldsymbol{x}-\boldsymbol{x}^{\prime}\right) \mathrm{d} x^{\prime} \mathrm{d} y^{\prime}
\end{aligned}
$$

or, equivalently, $\mathcal{R} f(\boldsymbol{x})=f_{1}(\boldsymbol{x})+j f_{2}(\boldsymbol{x})$, in accordance with (2). Note that this operator defines an allpass filter and that its impulse response is anti-symmetric: $\mathcal{R}\{\delta\}(\boldsymbol{x})=h_{\mathrm{R}}(\boldsymbol{x})=$ $-h_{\mathrm{R}}(-\boldsymbol{x})$.

\section{Properties of the Complex Riesz Operator}

We now present and discuss the key properties of $\mathcal{R}[14]$, [20].

Property 1 (Invariances): The complex Riesz operator is both translation- and scale-invariant

$$
\begin{aligned}
& \forall \boldsymbol{x}_{0} \in \mathbb{R}^{2}, \quad \mathcal{R}\left\{f\left(\cdot-\boldsymbol{x}_{0}\right)\right\}(\boldsymbol{x})=\mathcal{R}\{f(\cdot)\}\left(\boldsymbol{x}-\boldsymbol{x}_{0}\right) \\
& \forall a \in \mathbb{R}^{+}, \quad \mathcal{R}\{f(\cdot / a)\}(\boldsymbol{x})=\mathcal{R}\{f(\cdot)\}(\boldsymbol{x} / a) .
\end{aligned}
$$

The translation invariance directly follows from the definition while the scale-invariance is easily verified in the Fourier domain.

Property 2 (Steerability): The impulse response of the Riesz transform satisfies the rotation-covariance relation

$$
h_{\mathrm{R}}\left(\mathbf{R}_{\theta} \boldsymbol{x}\right)=e^{-j \theta} h_{\mathrm{R}}(\boldsymbol{x})
$$

where $\mathbf{R}_{\theta}=\left[\begin{array}{cc}\cos \theta & \sin \theta \\ -\sin \theta & \cos \theta\end{array}\right]$ is the matrix that implements a spatial rotation by the angle $\theta$.

This is established in the Fourier domain by showing that $\hat{h}_{\mathrm{R}}\left(\mathbf{R}_{\theta} \boldsymbol{\omega}\right)=e^{-j \theta} \hat{h}_{\mathrm{R}}(\boldsymbol{\omega})$.

The above result means that the Riesz transform can be rotated by simple multiplication with a complex number. An equivalent interpretation is that the component filters of the Riesz operator form a steerable ${ }^{3}$ filterbank in the sense defined by Freeman and Adelson [26]. This becomes most apparent when we link the operator to the following directional version of the Hilbert transform

$$
\mathcal{H}_{\theta} f(\boldsymbol{x})=\cos \theta f_{1}(\boldsymbol{x})+\sin \theta f_{2}(\boldsymbol{x})=\operatorname{Re}\left(e^{-j \theta} \mathcal{R} f(\boldsymbol{x})\right)
$$

whose impulse response corresponds to the rotated version of $h_{x}(\boldsymbol{x})=\operatorname{Re}\left(h_{\mathrm{R}}(\boldsymbol{x})\right)$. Note that the association between the Riesz and the directional Hilbert transforms is essentially the

\footnotetext{
${ }^{3}$ Steerability designates the property of being able to synthesize the response of any rotated version of a filter through an appropriate linear combination of basis functions.
}

same as the link between the gradient and the directional derivative (more on this later).

It is also instructive to determine the Riesz transform of a pure cosine function of frequency $\omega_{0}=\left(\omega_{0} \cos \theta_{0}, \omega_{0} \sin \theta_{0}\right)$. A simple Fourier-domain calculation yields

$$
\mathcal{R}\left\{\cos \left(\left\langle\boldsymbol{\omega}_{0}, \cdot\right\rangle\right)\right\}(\boldsymbol{x})=e^{j \theta_{0}} \sin \left(\left\langle\boldsymbol{\omega}_{0}, \boldsymbol{x}\right\rangle\right) .
$$

We can then apply (5) to recover a pure sine wave by steering the directional Hilbert transform in the appropriate direction

$$
\mathcal{H}_{\theta_{0}}\left\{\cos \left(\left\langle\boldsymbol{\omega}_{0}, \cdot\right\rangle\right)\right\}(\boldsymbol{x})=\sin \left(\left\langle\boldsymbol{\omega}_{0}, \boldsymbol{x}\right\rangle\right)
$$

This desirable "quadrature" behavior-as Larkin calls it [20] - follows from the fact that the central cut of the frequency response of $\mathcal{H}_{\theta}$ along the direction $\theta$ perfectly replicates the behavior of the 1-D Hilbert transform: $H_{\theta}(\omega \cos \theta, \omega \sin \theta)=-j \operatorname{sign}(\omega)$. The only limitation of the technique is that the ideal Hilbert-transform-like behavior falls off like $\cos \left(\theta-\theta_{0}\right)$, which calls for a precise adjustment of the analysis direction $\theta_{0}$.

Note that the above properties can also be stated using the more conventional vector formulation of the Riesz transform and that they are transposable to higher dimensions [14]. This is not so for the final one which is truly specific to the present 2-D complex formulation and central to our argumentation (cf. Section III-A).

Property 3 (Unitary Transform): The complex Riesz transform is a unitary mapping from $L_{2}\left(\mathbb{R}^{2}\right)$ into $L_{2}\left(\mathbb{R}^{2}\right)$ (Lebesgue's space of 2-D complex-valued finite energy functions).

This is equivalent to the property $\left|\hat{h}_{R}(\boldsymbol{\omega})\right|^{2}=1$ which is obviously satisfied. Thus, the adjoint of the Riesz operator is specified by

$$
\mathcal{R}^{*} f(x) \stackrel{\mathcal{F}}{\longleftrightarrow} \frac{\left(+j \omega_{x}+\omega_{y}\right)}{\|\boldsymbol{\omega}\|} \hat{f}(\boldsymbol{\omega})
$$

with the property that $\mathcal{R}^{-1}=\mathcal{R}^{*}$.

\section{Monogenic Signal and Directional Hilbert Analysis}

Given a 2-D signal $f(\boldsymbol{x}), \boldsymbol{x} \in \mathbb{R}^{2}$, Felsberg and Sommer define the three-component monogenic signal

$$
\mathbf{f}_{\mathrm{m}}(\boldsymbol{x})=(f(\boldsymbol{x}), \operatorname{Re}(\mathcal{R} f(\boldsymbol{x})), \operatorname{Im}(\mathcal{R} f(\boldsymbol{x})))=\left(f, f_{1}, f_{2}\right) .
$$

The local amplitude of the signal is given by $A(\boldsymbol{x})=\left\|\mathbf{f}_{\mathrm{m}}(\boldsymbol{x})\right\|=$ $\sqrt{f^{2}+f_{1}^{2}+f_{2}^{2}}$, while its local orientation $\theta$ and instantaneous phase $\xi$ are specified by the following relations:

$$
f=A \cos \xi, f_{1}=A \sin \xi \cos \theta, \quad f_{2}=A \sin \xi \sin \theta .
$$

As an alternative to the elaborate quaternion formalism proposed by Felsberg and Sommer, we prefer to view the monogenic signal analysis as a directional transposition of the 1-D analytic signal analysis (1). Specifically, we define $r=\sqrt{f_{1}^{2}+f_{2}^{2}}=|\mathcal{R} f|$ and interpret this quantity as the maximum response of the directional Hilbert transform operator; i.e., $r(\boldsymbol{x})=\max _{\theta}\left\{\mathcal{H}_{\theta} f(\boldsymbol{x})\right\}=\max _{\theta}\left\{\operatorname{Re}\left(e^{-j \theta} \mathcal{R} f(\boldsymbol{x})\right)\right\}$. 
This maximum response occurs in the direction specified by $\boldsymbol{u}=(\cos \theta, \sin \theta)=\left(f_{1} / r, f_{2} / r\right)$, as a direct consequence of (5). Next, we consider the complex variable $z=f+j r=A e^{j \xi}=A \cos \xi+j A \sin \xi$, which is then treated as if it was a 1-D entity. In particular, we recover the instantaneous amplitude $A=\sqrt{f^{2}+r^{2}}$ and phase $\xi=\arctan (r / f)$ of the signal $f$ along the direction $\boldsymbol{u}$. Likewise, we estimate the instantaneous frequency along the direction $\boldsymbol{u}$ by $\nu=\mathrm{D}_{\boldsymbol{u}} \xi=\langle\nabla \xi, \boldsymbol{u}\rangle$ where $\mathrm{D}_{\boldsymbol{u}}$ and $\nabla$ denote the directional derivative and gradient operators, respectively.

The conceptual advantage of viewing the monogenic signal analysis this way is that one can easily extend it to any other direction $\boldsymbol{u}^{\prime}=\left(\cos \theta^{\prime}, \sin \theta^{\prime}\right)$ by taking $r(\boldsymbol{x})=\operatorname{Re}\left(e^{-j \theta^{\prime}} \mathcal{R} f(\boldsymbol{x})\right)=\left\langle\left(f_{1}, f_{2}\right), \boldsymbol{u}^{\prime}\right\rangle$. In particular, we may select a direction of analysis that is associated with an area of interest, rather than a point location. This is justifiable when the signal is locally coherent and may be a good strategy for adding robustness to the orientation determination.

\section{E. Improved Tensor-Based Estimation of the Local Orientation}

The practical scheme that we propose is inspired by the structure tensor formalism [27]. In accordance with Felsberg's monogenic signal analysis, we select the orientation that maximizes $\mathcal{H}_{\theta} f(\boldsymbol{x})$, but instead of doing it pointwise, we optimize the response over a local neighborhood specified by a symmetric, positive weighting function $v(\boldsymbol{x})=v(-\boldsymbol{x}) \geq 0$ centered on the current position. Specifically, we determine the local orientation at $\boldsymbol{x}_{0}$ by solving the optimization problem

$$
\theta_{v}\left(\boldsymbol{x}_{0}\right)=\arg \max _{\theta \in[-\pi, \pi]} \int_{\mathbb{R}^{2}} v\left(\boldsymbol{x}-\boldsymbol{x}_{0}\right)\left|\mathcal{H}_{\theta} f(\boldsymbol{x})\right|^{2} \mathrm{~d} x \mathrm{~d} y .
$$

To simplify the derivation, we introduce the weighted innerproduct

$$
\langle f, g\rangle_{v}=\int_{\mathbb{R}^{2}} v(\boldsymbol{x}) f(\boldsymbol{x}) g(\boldsymbol{x}) \mathrm{d} x \mathrm{~d} y
$$

and rewrite the criterion to maximize as

$$
\begin{aligned}
& \int_{\mathbb{R}^{2}} v\left(\boldsymbol{x}-\boldsymbol{x}_{0}\right)\left|\mathcal{H}_{\theta} f(\boldsymbol{x})\right|^{2} \mathrm{~d} x \mathrm{~d} y \\
& =\left\|f_{1}\left(\cdot+\boldsymbol{x}_{0}\right) \cos \theta+f_{2}\left(\cdot+\boldsymbol{x}_{0}\right) \sin \theta\right\|_{v}^{2} \\
& =\left(\begin{array}{ll}
\cos \theta & \sin \theta
\end{array}\right) \mathbf{J}\left(\boldsymbol{x}_{0}\right)\left(\begin{array}{c}
\cos \theta \\
\sin \theta
\end{array}\right)
\end{aligned}
$$

where $\mathbf{J}\left(\boldsymbol{x}_{0}\right)$ is the $2 \times 2$ symmetric matrix whose entries are given by

$$
\begin{aligned}
{\left[\mathbf{J}\left(\boldsymbol{x}_{0}\right)\right]_{m n} } & =\left\langle f_{m}\left(\cdot+\boldsymbol{x}_{0}\right), f_{n}\left(\cdot+\boldsymbol{x}_{0}\right)\right\rangle_{v} \\
& =\int_{\mathbb{R}^{2}} v\left(\boldsymbol{x}-\boldsymbol{x}_{0}\right) f_{m}(\boldsymbol{x}) f_{n}(\boldsymbol{x}) \mathrm{d} x \mathrm{~d} y
\end{aligned}
$$

with $m, n \in\{1,2\}$. Because of the underlying inner-product structure, $\mathbf{J}\left(\boldsymbol{x}_{0}\right)$ is guaranteed to be positive-definite; in fact, it is the Riesz-transform counterpart of the local structure tensor which is build around the gradient. The determination of the orientation, therefore, amounts to maximizing the quadratic form $\boldsymbol{u}^{T} \mathbf{J}\left(\boldsymbol{x}_{0}\right) \boldsymbol{u}$ under the constraint $\|\boldsymbol{u}\|=1$, which is a classical eigenvalue problem. The optimal direction is specified by the eigenvector $\boldsymbol{u}_{1}$ corresponding to the largest eigenvalue $\lambda_{\max }$ of
$\mathbf{J}\left(\boldsymbol{x}_{0}\right)$. This eigenvalue gives the magnitude of the maximum response. By combining it with the smaller eigenvalue, we obtain a coherency index

$$
0 \leq \chi=\frac{\lambda_{\max }-\lambda_{\min }}{\lambda_{\max }+\lambda_{\min }} \leq 1
$$

which provides us with a precious indication of the degree of directionality of the local neighborhood. The directional Hilbert transform analysis will obviously perform best when the local neighborhood has a structure that is predominantly 1-D (i.e., $\chi \approx 1$ ), which suggests using the coherency as a general reliability index.

In this paper, we will go one step further by transfering these notions to the wavelet domain, which essentially amounts to performing a directional Hilbert analysis on bandpass-filtered (and critically-sampled) versions of the signal. The remarkable aspect is that this can be done in a stable, reversible way using bona fide wavelet bases.

\section{COMPlex RiesZ-Laplace WaVElets}

Our method of construction is simple conceptually. We start with a wavelet basis that is essentially isotropic and then use the unitary Riesz transform mapping to construct a corresponding complex steerable wavelet basis. The technical difficulty is to specify a good primary decomposition that replicates the isotropic behavior of the Laplace operator; we show that this can be done using polyharmonic splines.

\section{A. General Method for Constructing Complex Wavelet Bases}

The interesting consequence of Property 3 is that the complex Riesz operator will automatically map any basis of $L_{2}\left(\mathbb{R}^{2}\right)$ into another one. Indeed, we have

$$
\begin{aligned}
\forall \varphi_{k}, \varphi_{l} \in L_{2}\left(\mathbb{R}^{2}\right), \quad\left\langle\varphi_{k}, \varphi_{l}\right\rangle_{L_{2}} & =\left\langle\mathcal{R}^{*} \mathcal{R} \varphi_{k}, \varphi_{l}\right\rangle_{L_{2}} \\
& =\left\langle\mathcal{R} \varphi_{k}, \mathcal{R} \varphi_{l}\right\rangle_{L_{2}} .
\end{aligned}
$$

What is of even greater interest to us is that it will transform any wavelet basis into another complex one, thanks to the translation- and scale-invariance properties. Specifically, let us consider a dual pair of real-valued wavelets $\psi$ and $\tilde{\psi}$ that generate a nonseparable biorthogonal basis of $L_{2}\left(\mathbb{R}^{2}\right)$ such that

$$
\forall f \in L_{2}\left(\mathbb{R}^{2}\right), \quad f(\boldsymbol{x})=\sum_{i \in \mathbb{Z}} \sum_{\boldsymbol{k} \in \mathbb{Z}^{2} \backslash 2 \mathbb{Z}^{2}}\left\langle f, \tilde{\psi}_{i, \boldsymbol{k}}\right\rangle_{L_{2}} \psi_{i, \boldsymbol{k}}(\boldsymbol{x})
$$

with the short-hand notation $\psi_{i, \boldsymbol{k}}(\boldsymbol{x})=2^{i} \psi\left(2^{i} \boldsymbol{x}-\boldsymbol{k} / 2\right)$. Then, the complex wavelets $\psi^{\prime}=\mathcal{R} \psi$ and $\tilde{\psi}^{\prime}=\mathcal{R} \tilde{\psi}$ also form a biorthogonal pair with the property that

$$
\forall g \in L_{2}\left(\mathbb{R}^{2}\right), \quad g(\boldsymbol{x})=\sum_{i \in \mathbb{Z}} \sum_{\boldsymbol{k} \in \backslash 2 \mathbb{Z}^{2}}\left\langle g, \tilde{\psi}_{i, \boldsymbol{k}}^{\prime}\right\rangle_{L_{2}} \psi_{i, \boldsymbol{k}}^{\prime}(\boldsymbol{x}) .
$$

The latter expansion is obtained by considering the complex signal $g=\mathcal{R}\left\{g_{1}+j g_{2}\right\}$ where the real-valued functions $g_{1}$ and $g_{2}$ are both decomposed according to (10) and by making use of the unitary property (9). A crucial ingredient for this construction is Property 1 , which ensures that $\mathcal{R} \psi_{i, \boldsymbol{k}}=\psi_{i, \boldsymbol{k}}^{\prime}$ where $\psi^{\prime}=\mathcal{R} \psi$. 
If, in addition, the real wavelets $\psi$ or $\tilde{\psi}$ are isotropic, then we end up with a decomposition that is steerable and, therefore, rotation-invariant, thanks to Property 2 . Indeed, we can use (5) to rotate the wavelet corresponding to the real part of $\psi^{\prime}=\mathcal{R} \psi$ to any desired orientation:

$$
\operatorname{Re}\left(e^{-j \theta} \mathcal{R} \psi(\boldsymbol{x})\right)=\mathcal{H}_{\theta} \psi(\boldsymbol{x})=\operatorname{Re}\left(\psi^{\prime}\left(\mathbf{R}_{\theta} \boldsymbol{x}\right)\right)
$$

where the right-hand side of the equality is valid provided that the initial wavelet $\psi(\boldsymbol{x})=\psi(|\boldsymbol{x}|)$ is isotropic. The same argument obviously also holds if we substitute $\mathcal{R}$ by its adjoint $\mathcal{R}^{*}=\mathcal{R}^{-1}$.

While this complex-wavelet construction technique looks straightforward, at least formally, there are some technical difficulties associated with the specification of the corresponding complex multiresolution analysis of $L_{2}\left(\mathbb{R}^{2}\right)$ because the Riesz operator will not map a scaling function into another one; in particular, it will destroy the partition of unity and induce some delocalization due to the Fourier-domain singularity at the origin (suppression of the DC component). Fortunately for us, its effect is much less damaging on the wavelet side because of their vanishing moment property which tempers the singularity up to the order of the transform.

\section{B. Fractional Differential and Integral Operators}

The fractional Laplacian $(-\Delta)^{\alpha}$ with $\alpha \in \mathbb{R}^{+}$is the isotropic differential operator of order $2 \alpha$ whose Fourier-domain definition in the sense of distributions is

$$
(-\Delta)^{\alpha} f(\boldsymbol{x}) \stackrel{\mathcal{F}}{\longleftrightarrow}\|\boldsymbol{\omega}\|^{2 \alpha} \hat{f}(\boldsymbol{\omega})
$$

For $\alpha=n \in \mathbb{N}$, it is a purely local operator that is proportional to the classical $n$-fold Laplacian. Otherwise, its effect is nonlocal and described by the following distributional impulse response (cf. [28])

$$
\frac{C_{2 \alpha, d}}{\|\boldsymbol{x}\|^{2 \alpha+d}} \stackrel{\mathcal{F}}{\longleftrightarrow}\|\boldsymbol{\omega}\|^{2 \alpha}
$$

where $d=2$ is the number of dimensions and $C_{s, d}=$ $2^{s} \Gamma((d+s) / 2) /\left(\pi^{d / 2} \Gamma(-s / 2)\right)$ is an appropriate normalization constant; $\Gamma(r)=(r-1)$ ! is Euler's gamma function. Interestingly, the Fourier transform pair (13) is also valid for negative $\alpha$ provided that $-(\alpha+d / 2)$ is noninteger; otherwise, the left-hand side needs to be replaced by a term that is proportional to $\|x\|^{2 n} \log (\|x\|)$ with $n=-(\alpha+d / 2)$. This suggests extending the definition of the fractional Laplacian to negative orders as well, which leads to a fractional integral-like behavior. The so-defined family of fractional operators satisfies the composition rule $(-\Delta)^{\alpha_{1}}(-\Delta)^{\alpha_{2}}=(-\Delta)^{\alpha_{1}+\alpha_{2}}$ which follows from their Fourier-domain definition, with the convention that $(-\Delta)^{0}=$ Identity. Note that the fractional integral $(-\Delta)^{-\alpha} f$ with $\alpha>0$ is well-defined only for functions (or distributions) $f$ whose moments up to order $\lceil\alpha\rceil$ are zero, due to the singularity of the frequency response at the origin.

The operators $(-\Delta)^{1 / 2}$ and $(-\Delta)^{-1 / 2}$ are of special relevance because they allow us to make a direct link between the
Riesz transform and the complex-gradient (or Wirtinger) operator. Specifically, we have that

$$
\begin{aligned}
\left(\frac{\partial}{\partial x}+j \frac{\partial}{\partial y}\right) f(\boldsymbol{x}) & =-\mathcal{R}(-\Delta)^{\frac{1}{2}} f(\boldsymbol{x}) \\
& =\mathcal{R}\left(\frac{1}{2 \pi\|\boldsymbol{x}\|^{3}} * f\right)(\boldsymbol{x})
\end{aligned}
$$

where the first equality is trivially checked in the Fourier domain. The converse relation is

$$
\begin{aligned}
\mathcal{R} f(\boldsymbol{x}) & =-\left(\frac{\partial}{\partial x}+j \frac{\partial}{\partial y}\right)(-\Delta)^{-\frac{1}{2}} f(\boldsymbol{x}) \\
& =-\left(\frac{\partial}{\partial x}+j \frac{\partial}{\partial y}\right)\left(\frac{1}{2 \pi\|\boldsymbol{x}\|} * f\right)(\boldsymbol{x})
\end{aligned}
$$

where we are assuming that $f$ is zero-mean to avoid any ill-posedness problem. The latter formula allows us to interpret the Riesz transform as a smoothed version of the image gradient. Interestingly, the underlying smoothing kernel, which corresponds to a radial filtering with $\|\boldsymbol{\omega}\|^{-1}$, is the same as the one encountered in tomography when the reconstruction is performed by simple backprojection [29], [30]. This points towards a mathematical connection between the Radon and Riesz transforms, which is discussed in [11]; recently, Wietzke and Sommer have exploited this link to derive higher-order signal descriptors by iterating the transform [31].

The directional versions of relations (14) and (14) are

$$
\begin{aligned}
& \mathrm{D}_{\boldsymbol{u}} f(\boldsymbol{x})=-\mathcal{H}_{\theta}(-\Delta)^{\frac{1}{2}} f(\boldsymbol{x}) \\
& \mathcal{H}_{\theta} f(x)=-\mathrm{D}_{\boldsymbol{u}}(-\Delta)^{-\frac{1}{2}} f(\boldsymbol{x})
\end{aligned}
$$

where $\mathrm{D}_{\boldsymbol{u}} f=\langle\nabla f, \boldsymbol{u}\rangle$ is the derivative of $f$ along the direction $\boldsymbol{u}=(\cos \theta, \sin \theta)$. This is consistent with the $1-\mathrm{D}$ interpretation of the Hilbert transform being a lowpass-filtered version of the derivative operator.

\section{Polyharmonic Splines}

The polyharmonic splines are the spline functions associated with the fractional Laplacian operators. To introduce them, we consider the cardinal setting where the spline knots are on the cartesian grid $\mathbb{Z}^{2}$.

Definition 1: The continuously-defined function $s(\boldsymbol{x}) \in$ $L_{2}\left(\mathbb{R}^{2}\right)$ is a cardinal polyharmonic spline of order $\gamma$ iff. (cf. [32])

$$
(-\Delta)^{\gamma / 2} f(\boldsymbol{x})=\sum_{\boldsymbol{k} \in \mathbb{Z}^{2}} d[\boldsymbol{k}] \delta(\boldsymbol{x}-\boldsymbol{k})
$$

where $\delta(\boldsymbol{x})$ is the 2-D Dirac distribution.

The interpretation is that these splines have singularities of order $\gamma$ at the integers and that they have one degree of freedom per grid point. We can formally integrate the above equation by applying the inverse operator $(-\Delta)^{-\gamma / 2}$ whose impulse response is denoted by $\rho_{\gamma}(\boldsymbol{x})$. This leads to the following characterization of the space of cardinal polyharmonic splines:

$$
\mathcal{V}_{0}=\operatorname{span}\left\{\rho_{\gamma}(\boldsymbol{x}-\boldsymbol{k})\right\}_{\boldsymbol{k} \in \mathbb{Z}^{2}} \cap L_{2}\left(\mathbb{R}^{2}\right)
$$


which involves the integer shifts of the Green function of the defining operator; i.e., $(-\Delta)^{\gamma / 2} \rho_{\gamma}=\delta$. A more practical Shannon-like representation of these splines is

$$
\mathcal{V}_{0}=\left\{s(\boldsymbol{x})=\sum_{\boldsymbol{k} \in \mathbb{Z}^{2}} s[\boldsymbol{x}] \phi_{\gamma}(\boldsymbol{x}-\boldsymbol{k})\right\}
$$

where $s[\boldsymbol{x}]=\left.s(\boldsymbol{x})\right|_{\boldsymbol{x}=\boldsymbol{k}}$ are the integer samples of $s(\boldsymbol{x})$ and where $\phi_{\gamma}(\boldsymbol{x})$ is the unique cardinal polyharmonic spline interpolator such that $\phi_{\gamma}(\boldsymbol{k})=\delta[\boldsymbol{k}]$ (Kronecker delta). This basis function is best characterized by its Fourier transform

$$
\begin{aligned}
\phi_{\gamma}(\boldsymbol{x}) \stackrel{\mathcal{F}}{\longleftrightarrow} \hat{\phi}_{\gamma}(\boldsymbol{\omega}) & =\frac{\|\boldsymbol{\omega}\|^{-\gamma}}{\sum_{\boldsymbol{k} \in \mathbb{Z}^{2}}\|\boldsymbol{\omega}+2 \pi \boldsymbol{k}\|^{-\gamma}} \\
& =\frac{1}{1+\sum_{\boldsymbol{k} \in \mathbb{Z}^{2} \backslash\{\boldsymbol{0}\}}\left(\frac{\|\boldsymbol{\omega}\|}{\|\boldsymbol{\omega}+2 \pi \boldsymbol{k}\|}\right)^{\gamma}} .
\end{aligned}
$$

It is endowed with the following remarkable properties which can all be inferred from the above definition [32].

- $\phi_{\gamma}$ is a polyharmonic spline of order $\gamma$. This simply follows from the fact that $\|\omega\|^{\gamma} \hat{\phi}_{\gamma}(\omega)$ [the Fourier transform of $(-\Delta)^{\gamma / 2} \phi_{\gamma}$ ] is $2 \pi$-periodic.

- $\phi_{\gamma}$ is interpolating, which is equivalent to $\sum_{\boldsymbol{k} \in \mathbb{Z}^{2}} \hat{\phi}_{\gamma}(\boldsymbol{\omega}+$ $2 \pi \boldsymbol{k})=1$ (through Poisson's summation formula).

- Its Fourier transform is bounded $\left(0 \leq \hat{\phi}_{\gamma}(\boldsymbol{\omega}) \leq 1\right)$ and decaying away from the origin like $\mathcal{O}\left(\|\omega\|^{\gamma}\right)$. In particular, this implies that $\phi_{\gamma}$ has up to $\gamma-1$ derivatives in the $L_{2}$-sense.

- $\phi_{\gamma}$ generates a Riesz ${ }^{4}$ basis for the space of cardinal polyharmonic splines $\mathcal{V}_{0}$ provided that the order $\gamma>1$ [33]-[35].

- $\phi_{\gamma}$ has approximation order $\gamma$ and has the ability to interpolate all polynomials of total degree less than $\gamma([36]$, Chapter 4). This follows from the property that $\hat{\phi}_{\gamma}(\boldsymbol{\omega})$ has zeros of order $\gamma$ at $\boldsymbol{\omega}=2 \pi \boldsymbol{k}, \boldsymbol{k} \in \mathbb{Z}^{2} \backslash\{\boldsymbol{0}\}$ (Strang-Fix conditions of order $\gamma$ ).

- $\phi_{\gamma}(\boldsymbol{x})$ decays like $\mathcal{O}\left(\|\boldsymbol{x}\|^{-(\gamma+2)}\right)$ when $\gamma$ is noneven ([36], Theorem 4.3), and exponentially-fast otherwise $\left(\gamma \in 2 \mathbb{N}^{+}\right)$[32], [37].

- $\hat{\phi}_{\gamma}(\boldsymbol{\omega})=1+\mathcal{O}\left(\|\boldsymbol{\omega}\|^{\gamma}\right)$ as $\boldsymbol{\omega} \rightarrow \mathbf{0}$, meaning that it has a $\gamma$ th order of flatness at the origin (similar to a classical Butterworth filter of order $\gamma$ ).

- $\phi_{\gamma}(\boldsymbol{x})$ is symmetric and converges to the separable sinc interpolator $\operatorname{sinc}(\boldsymbol{x})$ as the order $\gamma$ goes to infinity [33].

\section{Laplacian-Like Wavelets}

Following Bacchelli et al. [35], we define the symmetric, Laplacian-like spline wavelet of (fractional) order $\gamma>1 / 2$

$$
\psi(\boldsymbol{x})=(-\Delta)^{\gamma / 2} \phi_{2 \gamma}(\mathbf{D} \boldsymbol{x})
$$

where $\phi_{2 \gamma}(\boldsymbol{x})$ is the polyharmonic spline interpolator of order $2 \gamma$ [cf. (17)] and where $\mathbf{D}$ is a given admissible 2-D dilation

\footnotetext{
${ }^{4} \mathrm{~A}$ Riesz basis is a fundamental concept in functional analysis that was introduced by the Hungarian mathematician Frigyes Riesz; it has not much to do with the Riesz transform that is due to Marcel Riesz (Frigyes' younger brother), except perhaps that the complex version of the Riesz transform maps a Riesz basis into another one.
}

(or subsampling) matrix (e.g., cartesian or quincunx). We then specify the basis functions

$$
\psi_{i, \boldsymbol{k}}(\boldsymbol{x})=|\operatorname{det}(\mathbf{D})|^{i / 2} \psi\left(\mathbf{D}^{i} \boldsymbol{x}-\mathbf{D}^{-1} \boldsymbol{k}\right)
$$

which are dilated (scale index $i$ ) and translated versions (space index $\boldsymbol{k}$ ) of one another. For a given $i$, these functions are centered on the rescaled lattice $\mathbf{D}^{-(i+1)} \mathbb{Z}^{2}$ which also corresponds to the location of the spline knots. Observe that the corresponding lattice is an upsampled version of the canonical grid $\mathbf{D}^{-i} \mathbb{Z}^{2}$ which is included as a subset.

Using such (normalized) wavelets, we specify the wavelet subspace at resolution $i$

$$
\mathcal{W}_{i}=\operatorname{span}\left\{\psi_{i, \boldsymbol{k}}(\boldsymbol{x})\right\}_{\boldsymbol{k} \in \mathbb{Z}^{2} \backslash \mathbf{D} \mathbb{Z}^{2}}
$$

which involves all wavelets at scale $i$ except the ones that are located on the canonical lattice $\mathbf{D}^{-i} \mathbb{Z}^{2}$. Note that this is a special instance of a nonseparable wavelet construction in which the usual $M=\operatorname{det}(\mathbf{D})-1$ wavelet channels at scale $i$ involve basis functions that are all identical up to a shift. Likewise, we introduce the scaling functions at resolution $i$

$$
\varphi_{i, \boldsymbol{k}}(\boldsymbol{x})=|\operatorname{det}(\mathbf{D})|^{i / 2} \phi_{\gamma}\left(\mathbf{D}^{i} \boldsymbol{x}-\boldsymbol{k}\right)
$$

and specify the corresponding approximation space

$$
\mathcal{V}_{i}=\operatorname{span}\left\{\varphi_{i, \boldsymbol{k}}(\boldsymbol{x})\right\}_{\boldsymbol{k} \in \mathbb{Z}^{2}}
$$

which is the subspace of polyharmonic splines with knots on the grid at resolution $i$ : $\mathbf{D}^{-i} \mathbb{Z}^{2}$.

The remarkable property of this construction is that the subspaces $\mathcal{V}_{i}$ and $\mathcal{W}_{i}$ are orthogonal and that their direct sum yields the finer resolution space: $\mathcal{V}_{i+1}=\mathcal{W}_{i} \oplus \mathcal{V}_{i}$; that is, the subspace of polyharmonic splines of order $\gamma$ with knots on the lattice $\mathbf{D}^{-(i+1)} \mathbb{Z}^{2}$. This is best understood by focusing on the wavelets at resolution $i=-1$.

Proposition 1: The wavelets $\psi_{-1, \boldsymbol{k}}(\boldsymbol{x})=$ $|\operatorname{det}(\mathbf{D})|^{-1 / 2}(-\Delta)^{\gamma / 2} \phi_{2 \gamma}(\boldsymbol{x}-\boldsymbol{k})$ are cardinal polyharmonic splines of order $\gamma$ (i.e., with knots on the lattice $\mathbb{Z}^{2}$ ). Moreover, they are orthogonal to $\mathcal{V}_{-1}$ whenever $\boldsymbol{k} \notin \mathbf{D} \mathbb{Z}^{2}$.

Proof: To establish the first part, we simply evaluate the Fourier transform of $(-\Delta)^{\gamma / 2} \psi_{-1, \boldsymbol{k}}(\boldsymbol{x})$

$$
\|\boldsymbol{\omega}\|^{\gamma} \underbrace{\|\boldsymbol{\omega}\|^{\gamma} \hat{\phi}_{2 \gamma}(\boldsymbol{\omega}) e^{-j\langle\boldsymbol{\omega}, \boldsymbol{k}\rangle}}_{\hat{\psi}_{-1, \boldsymbol{k}}(\boldsymbol{\omega})}=\frac{e^{-j\langle\boldsymbol{\omega}, \boldsymbol{k}\rangle}}{\sum_{\boldsymbol{k} \in \mathbb{Z}^{d}}\|\boldsymbol{\omega}+2 \pi \boldsymbol{k}\|^{-2 \gamma}}
$$

which is clearly $2 \pi$-periodic. This proves that $\psi_{-1, \boldsymbol{k}}(\boldsymbol{x})$ is a cardinal polyharmonic spline of order $\gamma$. For the second part, we use the property that $\mathcal{V}_{-1}=\operatorname{span}\left\{\rho_{\gamma}\left(\boldsymbol{x}-\mathbf{D} \boldsymbol{k}^{\prime}\right)\right\}_{\boldsymbol{k}^{\prime} \in \mathbb{Z}^{2}} \cap L_{2}\left(\mathbb{R}^{2}\right)$ where $\rho_{\gamma}$ is the Green function of $(-\Delta)^{\gamma / 2}$. We then perform the inner product manipulation

$$
\begin{aligned}
\mid \operatorname{det} & \left.(\mathbf{D})\right|^{\frac{1}{2}}\left\langle\psi_{-1, \boldsymbol{k}}, \rho_{\gamma}\left(\cdot-\mathbf{D} \boldsymbol{k}^{\prime}\right)\right\rangle \\
& =\left\langle(-\Delta)^{\frac{1}{2}} \phi_{2 \gamma}(\cdot-\boldsymbol{k}), \rho_{\gamma}\left(\cdot-\mathbf{D} \boldsymbol{k}^{\prime}\right)\right\rangle \\
& =\left\langle\phi_{2 \gamma}(\boldsymbol{x}-\boldsymbol{k}),(-\Delta)^{\frac{1}{2}} \rho_{\gamma}\left(\boldsymbol{x}-\mathbf{D} \boldsymbol{k}^{\prime}\right)\right\rangle \quad \text { (by duality) } \\
& =\left\langle\phi_{2 \gamma}(\cdot-\boldsymbol{k}), \delta\left(\cdot-\mathbf{D} \boldsymbol{k}^{\prime}\right)\right\rangle
\end{aligned}
$$

(Green function property)

$$
=\left\langle\phi_{2 \gamma}(\cdot), \delta\left(\cdot+\boldsymbol{k}-\mathbf{D} \boldsymbol{k}^{\prime}\right)\right\rangle=\delta\left[\boldsymbol{k}-\mathbf{D} \boldsymbol{k}^{\prime}\right] \text {. }
$$


Since $\phi_{2 \gamma}$ is interpolating and $\boldsymbol{k}-\mathbf{D} \boldsymbol{k}^{\prime}$ is necessarily integer, the expression vanishes except when $\boldsymbol{k}=\mathbf{D} \boldsymbol{k}^{\prime}$.

This combined with the fact that the polyharmonic splines for $\gamma>1 / 2$ provide a valid multiresolution analysis of $L_{2}\left(\mathbb{R}^{2}\right)$ yields the theorem below, which is a slight extension of previously published results. Specifically, Bacchelli et al. [35], [38] investigated the dyadic, nonfractional case (i.e., $\mathbf{D}=2 \mathbf{I}$ and $\gamma \in \mathbb{N}^{+}$), while Van De Ville et al. [34] fully characterized the underlying fractional wavelet bases, but in the quincunx case only (i.e., $\mathbf{D}=2 \mathbf{I}$ ).

Theorem 1: The polyharmonic spline wavelet $\psi(\boldsymbol{x})$ given by (18) generates a Riesz basis for $\mathcal{W}_{0}$. Moreover,

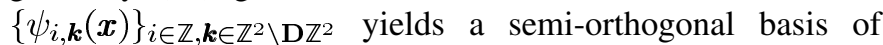
$L_{2}\left(\mathbb{R}^{2}\right)$ for all (fractional) orders $\gamma>1 / 2$.

The direct implication is that any function $f \in L_{2}\left(\mathbb{R}^{2}\right)$ has the following stable wavelet decompositions:

$$
\begin{aligned}
f(\boldsymbol{x}) & =\sum_{i \in \mathbb{Z}} \sum_{\boldsymbol{k} \in \mathbb{Z}^{2} \backslash \mathbf{D} \mathbb{Z}^{2}}\left\langle f, \tilde{\psi}_{i, \boldsymbol{k}}\right\rangle \psi_{i, \boldsymbol{k}}(\boldsymbol{x}) \\
& =\sum_{i \in \mathbb{Z}} \sum_{\boldsymbol{k} \in \mathbb{Z}^{2} \backslash \mathbf{D} \mathbb{Z}^{2}}\left\langle f, \psi_{i, \boldsymbol{k}}\right\rangle \tilde{\psi}_{i, \boldsymbol{k}}(\boldsymbol{x})
\end{aligned}
$$

which are termed primal or dual depending on the type of reconstruction wavelets. The complementary wavelet functions $\tilde{\psi}_{i, \boldsymbol{k}} \in \mathcal{W}_{i}$ are the unique duals of $\psi_{i_{,} \boldsymbol{k}}$ in the sense that they satisfy the biorthogonality property: $\left\langle\tilde{\psi}_{i, \boldsymbol{k}}, \psi_{\boldsymbol{i}^{\prime}, \boldsymbol{k}^{\prime}}\right\rangle=\delta_{i-i^{\prime}, \boldsymbol{k}-\boldsymbol{k}^{\prime}}$ with $\boldsymbol{k} \in \mathbb{Z}^{2} \backslash \mathbf{D} \mathbb{Z}^{2}$. While the primal wavelets are generated from a single template, there are $M-1$ distinct generators for the dual ones, as is usually the case for any general multidimensional wavelet construction with $M=\operatorname{det}(\mathbf{D})$. Structurally, the wavelet decomposition/reconstruction algorithm corresponds to an $M$-channel perfect reconstruction filterbank. The dual wavelets are defined implicitly through the perfect reconstruction conditions in Mallat's fast filterbank algorithm [34], [35].

\section{E. Complex, Riesz-Laplace Wavelet Basis of $L_{2}\left(\mathbb{R}^{2}\right)$}

Having set the mathematical foundations, we can now apply the (adjoint) Riesz transform to the above polyharmonic wavelet basis to obtain a bona fide complex transform. The corresponding complex, Riesz-Laplace wavelet of order $\gamma>1 / 2$ is given by

$$
\psi^{\prime}(\boldsymbol{x})=\mathcal{R}^{*} \psi(\boldsymbol{x})=\mathcal{R}^{*}(-\Delta)^{\gamma / 2} \phi_{2 \gamma}(\mathbf{D} \boldsymbol{x}) .
$$

The reason for considering $\mathcal{R}^{*}$ instead of $\mathcal{R}$ will become clearer in Section IV; it does not fundamentally change anything, except for the sign of the real part of the transform. By referring back to the argument in Section III-A, we immediately obtain the following corollary of Theorem 1 .

Corollary 1: The complex Riesz-Laplace wavelet $\psi^{\prime}(\boldsymbol{x})=\mathcal{R}^{*} \psi$ given by (23) generates a Riesz basis for $\mathcal{W}_{0}^{\prime}=\mathcal{R}^{*} \mathcal{W}_{0}=\operatorname{span}\left\{\psi^{\prime}\left(\boldsymbol{x}-\mathbf{D}^{-1} \boldsymbol{k}\right)\right\}_{\boldsymbol{k} \in \mathbb{Z}^{2} \backslash \mathbf{D} \mathbb{Z}^{2}}$. Moreover, $\left\{\psi_{i, \boldsymbol{k}}^{\prime}(\boldsymbol{x})=|\operatorname{det}(\mathbf{D})|^{i / 2} \psi^{\prime}\left(\mathbf{D}^{i} \boldsymbol{x}-\mathbf{D}^{-1} \boldsymbol{k}\right)\right\}_{i \in \mathbb{Z}, \boldsymbol{k} \in \mathbb{Z}^{2} \backslash \mathbf{D} \mathbb{Z}^{2} \text { yields }}$ a complex Riesz basis of $L_{2}\left(\mathbb{R}^{2}\right)$ provided that $\gamma>1 / 2$.

Interestingly, these wavelets are special instances (up to some proportionality factor) of the complex rotation-covariant wavelets that we had identified and characterized mathematically in previous work [39], [40]. Our earlier formulation did not involve the Riesz transform but rather a combination of iterated Laplace and complex gradient (or Wirtinger) operators of the form

$$
\mathrm{L}_{\gamma, N}=\left(\frac{\partial}{\partial x}-j \frac{\partial}{\partial y}\right)^{N}(-\Delta)^{\frac{\gamma-N}{2}} .
$$

The link with (23) is obtained by making use of (14) which yields the equivalence with the case $N=1$

$$
\begin{aligned}
\psi^{\prime}(\boldsymbol{x}) & =\left(\frac{\partial}{\partial x}-j \frac{\partial}{\partial y}\right)(-\Delta)^{\gamma / 2-1} \phi_{2 \gamma}(\mathbf{D} \boldsymbol{x}) \\
& =\mathrm{L}_{\gamma, 1} \phi_{2 \gamma}(\mathbf{D} \boldsymbol{x}) .
\end{aligned}
$$

The advantage of the present formulation is that we have identified the unitary mapping that transforms the complex wavelets into the polyharmonic ones (and vice versa) which simplifies the theory considerably. In particular, this allows us to step through the mathematical properties of the polyharmonic spline wavelets and to transpose them almost literally to the complex case. The argument rests upon the invariance properties of the complex Riesz transform which preserve the wavelet character of the transform, as well as the structure of the underlying Gram matrix (inner products between basis functions).

Property 4: (Semi-Orthogonality): The polyharmonic spline and complex Riesz-Laplace wavelets are orthogonal across scales

$$
\begin{aligned}
\forall k, \boldsymbol{k}^{\prime} \in \mathbb{Z}^{2} \backslash \mathbf{D} \mathbb{Z}^{2}, \quad i \neq j \\
\left\langle\psi_{i, \boldsymbol{k}}, \psi_{j, \boldsymbol{k}^{\prime}}\right\rangle=0 \Leftrightarrow\left\langle\mathcal{R}^{*} \psi_{i, \boldsymbol{k}}, \mathcal{R}^{*} \psi_{j, \boldsymbol{k}^{\prime}}\right\rangle=0 .
\end{aligned}
$$

This follows from Proposition 1.

Property 5: (Smoothness): The critical Sobolev exponent of the polyharmonic spline and complex Riesz-Laplace wavelets of order $\gamma$ is $s_{\max }=\gamma-1$.

The smoothness (or degree of differentiability) of a wavelet is often characterized by its inclusion in the Sobolev spaces $W_{2}^{s}=\left\{f \in L_{2}\left(\mathbb{R}^{2}\right) \mid(-\Delta)^{s / 2} f \in L_{2}\left(\mathbb{R}^{2}\right)\right\}$ which represent the class of functions with $s$ derivatives in the $L_{2}$-sense. We know that $\psi \in W_{2}^{s}$ for $s<s_{\max }=\gamma-1$ by construction, since it is a polyharmonic spline of order $\gamma . \mathcal{R}^{*} \psi$ is included in the same Sobolev spaces as a direct consequence of the norm-preservation property of the complex Riesz transform; in particular, $\|\psi\|_{L_{2}}=\left\|\mathcal{R}^{*} \psi\right\|_{L_{2}}$ and $\left\|(-\Delta)^{s / 2} \psi\right\|_{L_{2}}=$ $\left\|(-\Delta)^{s / 2} \mathcal{R}^{*} \psi\right\|_{L_{2}}$.

Property 6: (Operator Like-Behavior): The polyharmonic spline wavelet transform implements a multiscale version of the Laplace operator

$$
\left\langle f, \psi\left(\mathbf{D}^{i} \cdot-\boldsymbol{x}\right)\right\rangle=(-\Delta)^{\gamma / 2}\left(f * \varphi\left(\mathbf{D}^{i} \cdot\right)\right)(\boldsymbol{x})
$$

where the smoothing kernel is $\varphi(\boldsymbol{x})=\phi_{2 \gamma}(\mathbf{D} \boldsymbol{x})$, while the Riesz-Laplace wavelet transform does the same for the Riesz transform of the input signal

$$
\begin{aligned}
& \left\langle f, \mathcal{R}^{*} \psi\left(\mathbf{D}^{i} \cdot-\boldsymbol{x}\right)\right\rangle \\
& \quad=\left\langle\mathcal{R} f, \psi\left(\mathbf{D}^{i} \cdot-\boldsymbol{x}\right)\right\rangle \\
& \quad=(-\Delta)^{\gamma / 2}\left(\mathcal{R} f * \varphi\left(\mathbf{D}^{i} \cdot\right)\right)(\boldsymbol{x}) .
\end{aligned}
$$


Property 7: The polyharmonic spline and complex Riesz-Laplace wavelet transforms with parameter $\gamma$ have approximation order $\gamma$. Specifically, this means that the error of the scale-truncated approximation of a function $f \in W_{2}^{\gamma}$ at resolution $i_{0}$ decays like the $\gamma$ th power of that scale

$$
\begin{aligned}
& \left\|\sum_{i=-\infty} \sum_{\boldsymbol{k} \in \mathbb{Z}^{2} \backslash \mathbf{D} \mathbb{Z}^{2}}\left\langle f, \tilde{\psi}_{i, \boldsymbol{k}}\right\rangle \psi_{i, \boldsymbol{k}}\right\|_{L_{2}} \\
& \quad \leq C_{\gamma}\left(2^{-i_{0}}\right)^{\gamma}\left\|(-\Delta)^{\frac{\gamma}{2}} f\right\|_{L_{2}} \\
& \left\|\sum_{i=-\infty}^{i_{0}-1} \sum_{\boldsymbol{k} \in \mathbb{Z}^{2} \backslash \mathbf{D} \mathbb{Z}^{2}}\left\langle f, \mathcal{R}^{*} \tilde{\psi}_{i, \boldsymbol{k}}\right\rangle \mathcal{R}^{*} \psi_{i, \boldsymbol{k}}\right\|_{L_{2}} \\
& \quad \leq C_{\gamma}\left(2^{-i_{0}}\right)^{\gamma}\left\|(-\Delta)^{\frac{\gamma}{2}} f\right\|_{L_{2}}
\end{aligned}
$$

where $C_{\gamma}$ is a constant. As $i_{0} \rightarrow+\infty$, the approximation error tends to zero which proves that the both wavelet representations are dense in $L_{2}\left(\mathbb{R}^{2}\right)$.

This is a standard approximation-theoretic result for the polyharmonic splines [32], [34], [35]. It carries over to the complex Riesz wavelets by considering the expansion of $\mathcal{R} f$ instead of $f$ and by noting that all $L_{2}$-norms in the above inequality remain unchanged if we replace $f$ by $\mathcal{R} f$ or $\mathcal{R}^{*} f$ (unitary property).

Property 8: (Vanishing moments): The polyharmonic spline and Riesz-Laplace wavelets of order $\gamma$ have $\lceil\gamma\rceil$ vanishing moments.

The vanishing-moments property for the polyharmonic spline wavelets follows from the fact that the interpolator $\phi_{\gamma}$ satisfies the Strang-Fix conditions of order $\gamma$ (cf. Section III-C). In the frequency domain, this translates into the vanishing of $\hat{\psi}(\boldsymbol{\omega})$ and of all its partial derivatives up to order $\lfloor\gamma\rfloor$ at the origin [41]. Applying the Riesz transform does not fundamentally affect this behavior because the frequency response is also vanishing at the origin. Another way to see this is to observe that $\hat{\psi}(\boldsymbol{\omega}) \propto\|\boldsymbol{\omega}\|^{\gamma}$ and $\mathcal{R}^{\hat{*}} \psi(\boldsymbol{\omega}) \propto\left(j \omega_{1}+\omega_{2}\right)\|\boldsymbol{\omega}\|^{\gamma-1}, \boldsymbol{\omega} \rightarrow \mathbf{0}$, as a consequence of the operator-like behavior in Property 6 .

Property 9: (Fast Implementation): The polyharmonic spline and complex Riesz-Laplace wavelet transforms are perfectly reversible. They both have a fast filterbank implementation.

This latter aspect is further developed in Section V-B.

\section{Monogenic WaVelet Analysis}

Referring back to Section II-D, we now show how to take advantage of the paired transforms to perform a wavelet-domain monogenic signal analysis. In essence, this amounts to performing the monogenic analysis of the bandpass-filtered signals $\left(f * \psi_{i}\right)(\boldsymbol{x})$ where $\psi_{i}(\boldsymbol{x})=|\operatorname{det}(\mathbf{D})|^{i / 2} \psi\left(\mathbf{D}^{i} \boldsymbol{x}\right)$ is the normalized and rescaled polyharmonic spline wavelet at scale $i$. Indeed, since the primary (real-valued) analysis wavelet $\psi_{i}(\boldsymbol{x})$ is symmetric, the relevant set of wavelet coefficients of the signal $f$ can be expressed as

$$
\begin{aligned}
w_{i}[\boldsymbol{k}] & =\left\langle f, \psi_{i, \boldsymbol{k}}\right\rangle \\
& =\left.\left(\psi_{i} * f\right)(\boldsymbol{x})\right|_{\boldsymbol{x}=\mathbf{D}^{-(i+1)} \boldsymbol{k}} \\
r_{1, i}[\boldsymbol{k}]+j r_{2, i}[\boldsymbol{k}] & =\left\langle f, \mathcal{R}^{*} \psi_{i, \boldsymbol{k}}\right\rangle \\
& =\left\langle\mathcal{R} f, \psi_{i, \boldsymbol{k}}\right\rangle \\
& =\left.\mathcal{R}\left(\psi_{i} * f\right)(\boldsymbol{x})\right|_{\boldsymbol{x}=\mathbf{D}^{-(i+1)} \boldsymbol{k}}
\end{aligned}
$$

where $r_{1, i}[\boldsymbol{k}]$ and $r_{2, i}[\boldsymbol{k}]$ are the real and imaginary parts of the complex-valued Riesz wavelet coefficients; the sampling lattice at resolution $i$ is $\mathbf{D}^{-(i+1)} \mathbb{Z}^{2}$ in accordance with our wavelet definition (19). The above equations provide an exact correspondence between the wavelet coefficient triples $\left(w_{i}[\boldsymbol{k}], r_{1, i}[\boldsymbol{k}], r_{2, i}[\boldsymbol{k}]\right)$ and the sample values of $\left(\psi_{i} * \mathbf{f}_{m}\right)(\boldsymbol{x})$ where $\mathbf{f}_{m}(\boldsymbol{x})$ is Felsberg's monogenic signal, as defined by (7). Concretely, this means that we can perform a full multiresolution monogenic signal analysis based on the above wavelet-domain description of the signal.

There are two complementary aspects in this analysis that we propose to handle separately: 1) the determination of the local orientation, and 2) the Hilbert analysis along the preferential orientation, which gives access to the local amplitude, phase and frequency information. The main point is that we can determine these quantities for each scale and location index $(i, \boldsymbol{k})$.

\section{A. Wavelet-Domain Orientation Analysis}

In accordance with the scheme outlined in Section II-E, our estimation of the orientation is based on the evaluation of the structure matrix $\mathbf{J}_{i}(\boldsymbol{k})$ associated with the wavelet cell $(i, \boldsymbol{k})$ and a given weighting sequence $v[\boldsymbol{l}] \geq 0$. The entries of this $2 \times 2$ symmetric matrix are given by

$$
\left[\mathbf{J}_{i}(\boldsymbol{k})\right]_{m n}=J_{m n}=\sum_{\boldsymbol{l} \in \mathbb{Z}^{2}} v[\boldsymbol{l}-\boldsymbol{k}] r_{m, i}[\boldsymbol{l}] r_{n, i}[\boldsymbol{l}]
$$

with $m, n \in 1,2$. The estimation neighborhood is specified by the weighting sequence $v[l]$, which is center-symmetric, normalized $\left(\sum_{\boldsymbol{k} \in \mathbb{Z}^{2}} v[\boldsymbol{k}]=1\right)$ and decreasing away from the origin. It is chosen to be quite localized (e.g., Gaussian window with $\sigma \in[1,2])$. The eigen-decomposition of this matrix yields the three primary directional wavelet features.

- Orientation:

$$
\theta=\frac{1}{2} \arctan \left(\frac{2 J_{12}}{J_{22}-J_{11}}\right) .
$$

- Hilbert-transform energy:

$$
\lambda_{\max }=\frac{1}{2}\left(J_{11}+J_{22}+\sqrt{\left(J_{22}-J_{11}\right)^{2}+4 J_{12}^{2}}\right) .
$$

- Coherency:

$$
\chi=\frac{\lambda_{\max }-\lambda_{\min }}{\lambda_{\max }+\lambda_{\min }}=\frac{\sqrt{\left(J_{22}-J_{11}\right)^{2}+4 J_{12}^{2}}}{J_{22}+J_{11}} .
$$

The angle $\theta$ gives the orientation along which the directional Hilbert transform of the bandpass-filtered signal is maximum on average within the local neighborhood specified by $v$. The extent of this response is measured by the Hilbert-transform energy. The coherency $0 \leq \chi \leq 1$ quantifies the degree of directionality of the signal over the neighborhood of interest. A value close to one indicates that the structure is predominantly 1-D, which reinforces the validity of the subsequent AM/FM analysis.

The local coherence hypothesis that is implicit in the analytic signal formalism is more questionable when the eigenvalues $\lambda_{\max }$ and $\lambda_{\min }$ are both large. This typically corresponds to the signature of a localized isotropic image structure (peak or blob), 


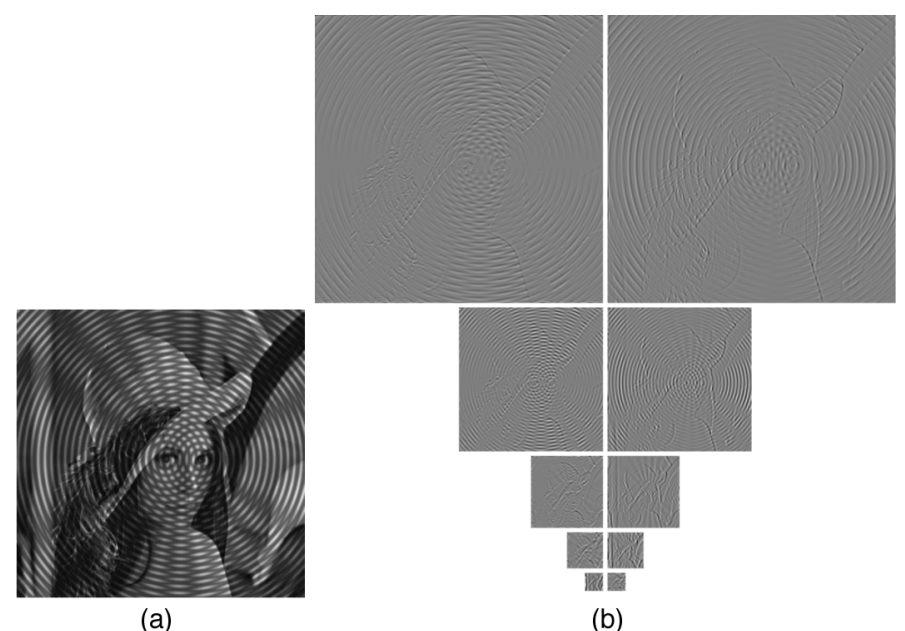

Fig. 1. "Psychedelic Lena": (a) "Lena" with two superimposed concentric spherical waves originating from the eyes; the propeller effect is due to the interference of the two waves. (b) Its complex Riesz wavelet transform with $\gamma=4$.
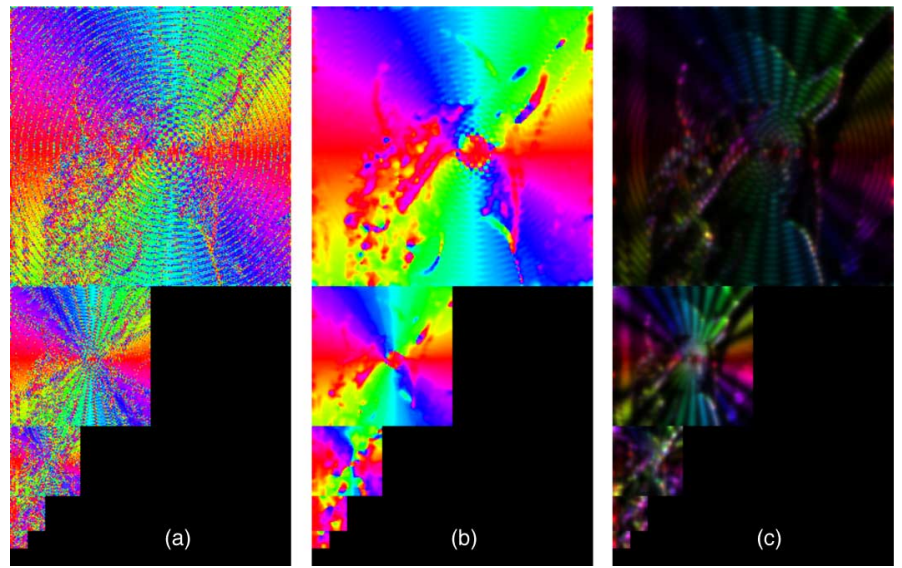

Fig. 2. Multiresolution directional analysis derived from the Riesz wavelet transform in Fig. 1(b) [the orientation is encoded in the hue channel]. (a) Pointwise orientation. (b) Tensor-based orientation with Gaussian observation window. (c) Combined visualization of tensor-based features: orientation in hue, coherency in saturation, and local Hilbert-transform energy in brightness.

or a corner, which may be identified using a Harris-like detector [27], [42]. A possible wavelet-domain corner index is

$$
h_{\text {corner }}=\lambda_{\max } \lambda_{\min }-\kappa\left(\lambda_{\max }+\lambda_{\min }\right)^{2}
$$

where $\kappa$ is an empirical constant in the range 0.04-0.5.

Note that a pointwise analysis can be performed by selecting the weighting sequence $v$ to be a unit impulse. In that case, the Hilbert-transform energy and orientation are given by the square modulus and the phase of $\left\langle f, \mathcal{R}^{*} \psi_{i, \boldsymbol{k}}\right\rangle$, respectively; the coherency and corner index are then meaningless because the rank of $\mathbf{J}_{i}[k]$ is necessarily one.

An example of directional wavelet analysis is presented in Figs. 1-2, using color coding. The scale progression is dyadic with a slightly redundant wavelet sampling (i.e., $\boldsymbol{k} \in \mathbb{Z}^{2}$ instead of $\boldsymbol{k} \in \mathbb{Z}^{2} \backslash 2 \mathbb{Z}^{2}$ ); the primary analysis wavelet $\psi$ is a Mexican-hat-like polyharmonic spline of order $\gamma=4$, as specified in Section V-A. The intent here is to demonstrate the difference between a pointwise orientation estimation with $v[\boldsymbol{k}]=\delta[\boldsymbol{k}]$ [cf. Fig. 2(a)] and a tensor-based analysis where $v$ is a fixed
Gaussian window with $\sigma=1.5$ [cf. Fig. 2(b)]. The latter orientation map (wrapped within the interval $[0, \pi]$ ) is less noisy; it is more robust and in better agreement with our visual perception of local directionality. Finally, in Fig. 2(c), we have combined the three primary directional wavelet features into a single image using the HSB channels: hue for orientation, saturation for squared coherency, and brightness for energy. Interestingly, the wave components, which have most of their energy in the second subband (scale $i=-2$ ), are clearly visible at that scale.

\section{B. Wavelet-Domain AM/FM Analysis}

The idea is now to perform an analytic signal analysis along the direction provided by the orientation analysis and encoded by the unit vector $\boldsymbol{u}=(\cos \theta, \sin \theta)$. The corresponding directional Hilbert component is given by

$$
\begin{aligned}
q_{i}[\boldsymbol{k}] & =\left\langle\mathcal{H}_{\theta} f, \psi_{i, \boldsymbol{k}}\right\rangle=\operatorname{Re}\left(\left\langle e^{-j \theta} \mathcal{R} f, \psi_{i, \boldsymbol{k}}\right\rangle\right) \\
& =\operatorname{Re}\left(e^{-j \theta}\left\langle f, \mathcal{R}^{*} \psi_{i, \boldsymbol{k}}\right\rangle\right) \\
& =r_{1, i}[k] \cos \theta+r_{2, i}[\boldsymbol{k}] \sin \theta .
\end{aligned}
$$


This leads to the specification of the analytical wavelet transform: $w_{i}[k]+j q_{i}[\boldsymbol{k}]=A_{i}[k] \cdot e^{j \xi}$, which is locally oriented in the preferential direction $\theta$. The wavelet-domain amplitude and local phase are computed as

$$
\begin{aligned}
A_{i}[\boldsymbol{k}] & =\sqrt{w_{i}^{2}[\boldsymbol{k}]+q_{i}[\boldsymbol{k}]^{2}} \\
\xi & =\arctan \left(\frac{q_{i}[\boldsymbol{k}]}{w_{i}[\boldsymbol{k}]}\right)
\end{aligned}
$$

where $q_{i}[\boldsymbol{k}]$ is given by (29) with $\theta$ specified by (26).

Recalling that the wavelet analysis at scale $i$ is equivalent to a bandpass filtering of the input signal, we may, therefore, use the wavelet amplitude to extract a putative AM signal component in the corresponding wavelet subband. The other useful information (FM) is carried by the instantaneous frequency (or wave number) that corresponds to the spatial derivative of $\xi$ in the direction $\boldsymbol{u}$. After some algebraic manipulations (cf. Appendix I), we obtain an explicit form for the instantaneous frequency:

$$
\begin{aligned}
\nu_{i}[\boldsymbol{k}]= & \left(-q_{i}[\boldsymbol{k}]\left(w_{x, i}[\boldsymbol{k}] \cos \theta+w_{y, i}[\boldsymbol{k}] \sin \theta\right)\right. \\
& \left.+w_{i}[\boldsymbol{k}]\left(r_{1 x, i}[\boldsymbol{k}]+r_{2 y, i}[\boldsymbol{k}]\right)\right) / A_{i}^{2}[\boldsymbol{k}], \\
= & \frac{-q_{i}[\boldsymbol{k}] \operatorname{Re}\left(e^{j \theta}\left\langle f, \mathcal{R}^{*} \psi_{i, \boldsymbol{k}}^{\prime}\right\rangle\right)-w_{i}[\boldsymbol{k}]\left\langle f, \psi_{i, \boldsymbol{k}}^{\prime}\right\rangle}{A_{i}^{2}[\boldsymbol{k}]}
\end{aligned}
$$

which has the advantage of avoiding the use of the arctan function as well as the problems associated with phase-wrapping. The additional required quantities are the spatial derivatives of $w, r_{1}$ and $r_{2}$. These are computed economically by considering the two auxiliary Riesz-Laplace wavelets $\psi^{\prime}$ and $\mathcal{R}^{*} \psi^{\prime}$ of reduced order $\gamma-1$, which are defined as

$$
\begin{aligned}
\psi^{\prime}(\boldsymbol{x})= & \left(-\frac{\partial}{\partial x}-j \frac{\partial}{\partial y}\right) \mathcal{R}^{*} \psi(\boldsymbol{x}) \\
= & (-\Delta)^{1 / 2} \psi(\boldsymbol{x}) \\
& \stackrel{\mathcal{F}}{\longrightarrow}\|\boldsymbol{\omega}\| \hat{\psi}(\boldsymbol{\omega}), \\
\mathcal{R}^{*} \psi^{\prime}(\boldsymbol{x})= & \left(\frac{\partial}{\partial x}-j \frac{\partial}{\partial y}\right) \psi(\boldsymbol{x}) \\
& \stackrel{\mathcal{F}}{\longleftrightarrow}\left(j \omega_{x}+\omega_{y}\right) \hat{\psi}(\boldsymbol{\omega}) .
\end{aligned}
$$

The first is real, symmetric, and is designed to calculate the Laplacian-like term $-\left(r_{1 x, i}[\boldsymbol{k}]+r_{2 y, i}[\boldsymbol{k}]\right)=\left\langle f, \psi_{i, \boldsymbol{k}}^{\prime}\right\rangle$, while the second is complex and is associated with the complex-gradient term $w_{x, i}[\boldsymbol{k}]-j w_{y, i}[\boldsymbol{k}]=\left\langle f, \mathcal{R}^{*} \psi_{i, \boldsymbol{k}}^{\prime}\right\rangle$. The main point is that these additional "derivative" wavelet transforms can be calculated using essentially the same filterbank algorithm with an appropriate modification of the analysis filters. This means that the cost for obtaining an instantaneous frequency estimate is about twice that of the original monogenic wavelet decomposition. The advantage of the present formulation is that the frequency formula (34) is exact and that it does not involve any finite difference approximation of the spatial derivatives.

\section{Practical ImPlementation AND Results}

We conclude the presentation by discussing some important practical issues and by presenting concrete analysis examples.

\section{A. Improving the Invariance Properties}

The monogenic wavelet transform that was introduced so far is mathematically elegant because it is tied to a basis. However, there is a price to pay for this property which is some lack of invariance. We will now show how to bypass this limitation.

1) Shift Invariance: Eventhough it is build around a single wavelet, the wavelet analysis of Section III is somewhat awkward to interpret because the transform is missing the wavelets at the subsampling locations corresponding to the next coarser grid; this is expressed mathematically in Theorem 1 by having the spatial wavelet index $(\boldsymbol{k})$ restricted to the set $\mathbb{Z}^{2} \backslash \mathbf{D} \mathbb{Z}^{2}$. For image analysis, it is tempting to include the "missing" shifts as well (i.e., consider the complete cartesian sampling set $\mathbb{Z}^{2}$ ), and to rearrange the wavelet coefficients into a single, imagelike subband at scale $i$. This is equivalent to not subsampling the wavelet subband after digital filtering. For instance, in the dyadic case where $\mathbf{D}=2 \mathbf{I}$, this description is associated with the enlarged analysis spaces

$$
\mathcal{W}_{i}^{+}=\operatorname{span}\left\{2^{i} \psi\left(2^{i} \boldsymbol{x}-\boldsymbol{k} / 2\right)\right\}_{\boldsymbol{k} \in \mathbb{Z}^{2}}
$$

where $\psi$ is the generating wavelet of order $\gamma$. The corresponding notation for the (nonsubsampled) wavelet coefficients at scale $i$ is $w_{i}[\mathbf{k}]$ (as before) and all previous formulas are still applicable with $\boldsymbol{k}$ spanning over $\mathbb{Z}^{2}$ instead of $\mathbb{Z}^{2} \backslash 2 \mathbb{Z}^{2}$. The same arrangement is also used for the Riesz branch of the transform. This extension improves the shift-invariance of the decomposition and its cost is moderate (total redundancy factor of $1 / 3$ ). With this sampling arrangement, the overall redundancy of the monogenic Riesz-Laplace transform is $R=3(1+(1 / 4)+$ $(1 / 16)+\cdots)=4$.

2) Rotation Invariance and Steerability: The other weak point is that the smoothing kernel $\phi_{2 \gamma}$ of our defining wavelet is not isotropic. In fact, it is sinc-like since it is an interpolating function. We propose to exploit the degrees of freedom provided by the enlarged wavelet spaces $\mathcal{W}_{i}^{+}$to tune the kernel's shape to make the wavelet more nearly isotropic. Our solution is to select the "isotropic" polyharmonic B-spline $\beta_{2 \gamma}$ as our new smoothing kernel [34]. This is justified by the fact that this $\mathrm{B}$-spline spans the same spline space as $\phi_{2 \gamma}$, with the advantage that is is better localized and that it converges to a Gaussian as $\gamma$ increases ([34], Proposition 2). The frequency-domain formula for our new smoothing kernel is

$$
\beta_{2 \gamma}(\boldsymbol{x}) \stackrel{\mathcal{F}}{\longleftrightarrow}\left(\frac{V\left(e^{j \boldsymbol{\omega}}\right)}{\|\boldsymbol{\omega}\|^{2}}\right)^{\gamma}
$$

where $V\left(e^{j \omega}\right)$ is the "most-isotropic" discrete Laplacian filter

$$
\begin{aligned}
V\left(e^{j \omega}\right)= & \frac{8}{3}\left(\sin ^{2}\left(\frac{\omega_{1}}{2}\right)+\sin ^{2}\left(\frac{\omega_{2}}{2}\right)\right) \\
& +\frac{2}{3}\left(\sin ^{2}\left(\frac{\omega_{1}+\omega_{2}}{2}\right)+\sin ^{2}\left(\frac{\omega_{1}-\omega_{2}}{2}\right)\right) .
\end{aligned}
$$

The corresponding mother wavelet to be used in replacement of the preceding one is $\psi_{\text {iso }}(\boldsymbol{x})=(-\Delta)^{\gamma / 2} \beta_{2 \gamma}(\mathbf{D} \boldsymbol{x})$, while the whole monogenic analysis procedure remains the same as described earlier. This is entirely justifiable mathematically since the modified Mexican-hat-like wavelet also spans the analysis 


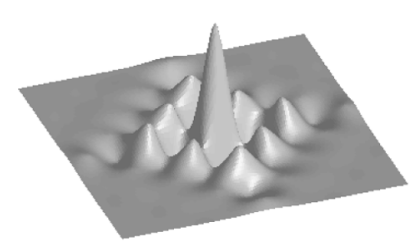

(a)

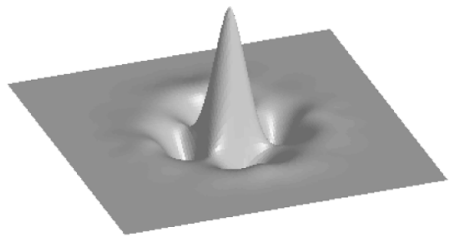

(b)
Fig. 3. Improving wavelet isotropy: (a) Primary polyharmonic spline wavelet basis generator $\psi(\boldsymbol{x})=(-\Delta)^{\gamma / 2} \phi_{2 \gamma}(2 \boldsymbol{x})$ with $\gamma=4$. (b) its Mexican-hatlike replacement $\psi_{\text {iso }}(\boldsymbol{x})=(-\Delta)^{\gamma / 2} \beta_{2 \gamma}(2 \boldsymbol{x})$, which spans the same space while being essentially isotropic.

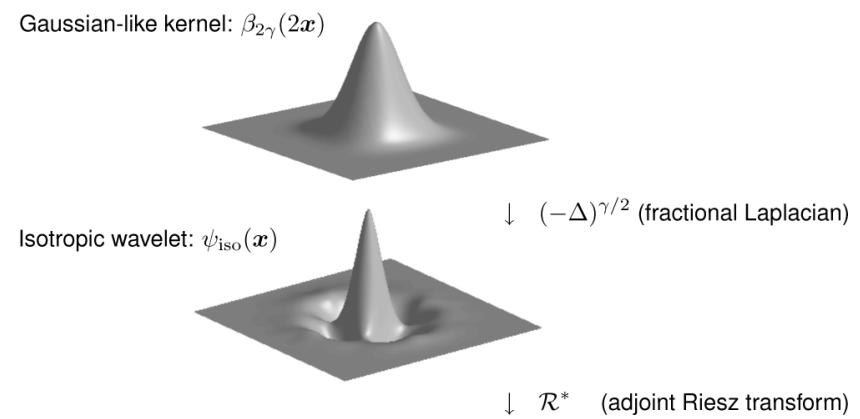

Complex Riesz wavelet: $\psi_{\text {Riesz }}(x)$
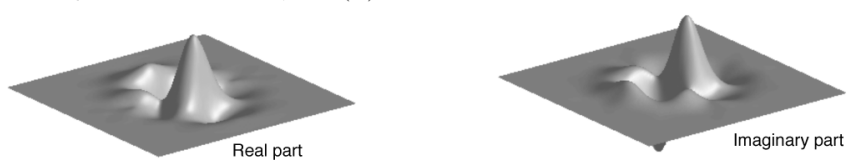

Fig. 4. Summary of relevant basis functions for the monogenic wavelet transform with $\gamma=4$. The real and imaginary parts of the complex Riesz wavelet are $90^{\circ}$-rotated versions of each other.

spaces $\mathcal{W}_{i}^{+}$. Concretely, this wavelet substitution amounts to a simple change of wavelet filters in the algorithm in a manner that is fully reversible and transparent to the user. This simple adaptation results into a dramatic improvement in wavelet isotropy, as illustrated in Fig. 3. The effect is equally helpful on the Riesz wavelet for it substantially improves the steerability of the transform; this is essential for the feature extraction process to be rotation-invariant.

A global summary and visualization of the relevant basis functions for monogenic wavelet analysis is provided in Fig. 4. The important point is that all basis functions are related to the smoothing kernel $\beta_{2 \gamma}(2 \boldsymbol{x})$ via some operator transformations (Laplacian and Riesz transform) that are truly scale and rotation-invariant. This means that we have a full control of isotropy (and steerability) through the shaping of the smoothing kernel. In the proposed B-spline scenario, this kernel is nearly isotropic and closely resembles a Gaussian with standard deviation $\sqrt{2 \gamma / 12}$.

The frequency responses of the analysis filterbank that implement the corresponding polyharmonic wavelet transform and its Riesz counterpart are shown in Fig. 5(a) and (b), respectively. The primary wavelet filters have a clear bandpass behavior and their response is very nearly isotropic, especially in the lower portion of the spectrum. The Riesz wavelet filters are directional along the horizontal or vertical directions. Due to the underlying

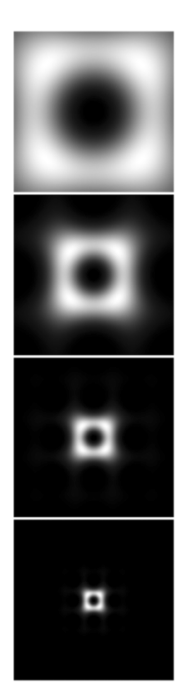

(a)

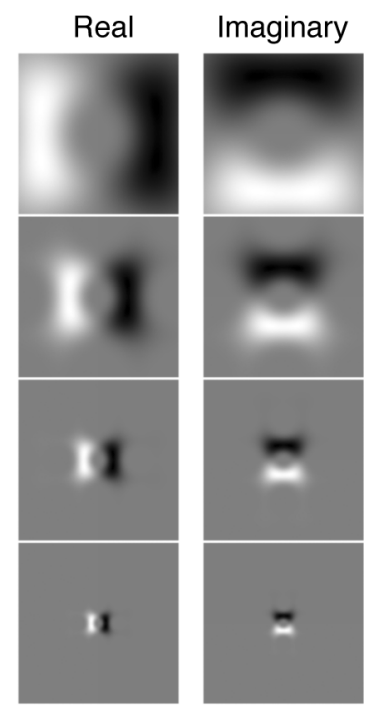

(b)
Fig. 5. Frequency responses of the monogenic wavelet analysis filters: (a) Mexican-hat-like wavelet $\hat{\psi}_{\text {iso, } i}(\omega)$ with $\gamma=4$, (b) corresponding Riesz wavelet $\widehat{\mathcal{R} *} \psi_{\text {iso, } i}(\omega)$. The scale $i$ is decreasing vertically from $i=0$ down to -3 .

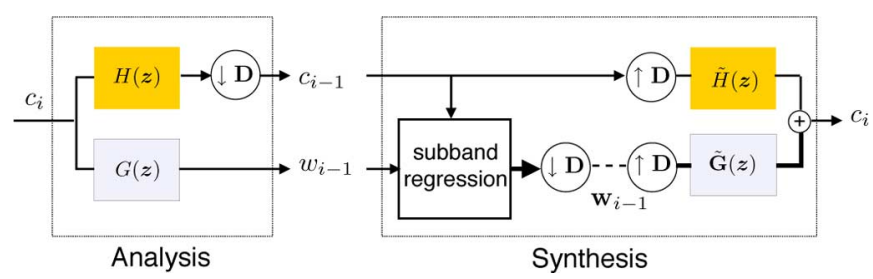

Fig. 6. Perfect reconstruction filterbank algorithm for the primary redundant wavelet transform. The role of the subband regression module is to convert the redundant wavelet representation into a nonredundant one described by the intermediate wavelet coefficient vector $\mathbf{w}_{i-1}$.

symmetries, the responses associated with the real and imaginary part of the transform are $90^{\circ}$-rotated versions of each other (in both space and frequency domains).

\section{B. Implementation}

The monogenic wavelet transform is implemented efficiently by running two perfect-reconstruction filterbanks in parallel: one for the primary (Mexican-hat-like) polyharmonic wavelet transform, and one for its Riesz counterpart which requires the use of complex arithmetics. We will now briefly describe the implementation of the primary wavelet transform, which corresponds to the block diagram in Fig. 6. The key components are as follows.

- The analysis part that consist of a lowpass channel (filter $H\left(\mathbf{z}^{-1}\right)$ ) and a single wavelet channel (highpass filter $\left.G\left(\mathbf{z}^{-1}\right)\right)$.

- A reconstruction stage that implements the dual frame operator. It includes a conventional lowpass channel (up-sampling and filtering by $\tilde{H}(\mathbf{z})$ ), a regression module which produces a critically-sampled output, and, finally, a standard wavelet reconstruction module (upsampling and multichannel filtering by $\tilde{\mathbf{G}}(z)$ ) whose input is the $N$-component wavelet coefficient vector $\mathbf{w}_{i-1}$ with $N=|\operatorname{det}(\mathbf{D})|-1$. 
The nonstandard aspect of the scheme is that there is no downsampling in the wavelet analysis channel, which produces a slightly redundant transform. The redundancy is removed during the reconstruction process by the subband regression module which outputs a sequence of critically-sampled wavelet coefficients $\mathbf{w}_{i-1}$ that is maximally consistent with $w_{i-1}$ in the least-squares sense. In effect, the algorithm projects the redundant wavelet analysis onto a nonredundant system (standard wavelet basis). Here, we will only specify the analysis part of the algorithm which is sufficient to characterize the process. For the synthesis part, we refer the reader to [43] which contains all the details for designing the complementary set of regression-based reconstruction filters.

Since we want the isotropic/monogenic wavelets to be applied on the analysis side, we choose to represent the successive multiresolution approximations of the input signal in terms of the dual basis function $\varphi(\boldsymbol{x})=\tilde{\beta}_{\gamma}(\boldsymbol{x})$

$$
f_{i}=\operatorname{Proj}_{V_{i}} f=\sum_{\boldsymbol{k} \in \mathbb{Z}^{2}} c_{i}[\boldsymbol{k}] \varphi_{i, \boldsymbol{k}} .
$$

We recall that $\tilde{\beta}_{\gamma}(x) \in V_{0}$ is the unique (biorthogonal) polyharmonic spline such that $\left\langle\tilde{\beta}_{\gamma}(\cdot-\boldsymbol{k}), \beta_{\gamma}\left(\cdot-\boldsymbol{k}^{\prime}\right)\right\rangle=\delta_{\boldsymbol{k}-\boldsymbol{k}^{\prime}}$.

The algorithm is initialized by assuming that the input signal is bandlimited; i.e., $f(\boldsymbol{x})=\sum_{\boldsymbol{k} \in \mathbb{Z}^{2}} f[k] \operatorname{sinc}(\boldsymbol{x}-\boldsymbol{k})$. The fine-scale coefficient sequence is, therefore, given by $c_{0}[\boldsymbol{k}]=\left\langle f, \beta_{\gamma}(\cdot-\boldsymbol{k})\right\rangle=\left(h_{\text {init }} * f\right)[\boldsymbol{k}]$ where $h_{\text {init }}[\boldsymbol{k}]=\left\langle\right.$ sinc, $\left.\beta_{\gamma}(\cdot-\boldsymbol{k})\right\rangle$ is the optimal projection prefilter. The coarsening part of the decomposition is achieved by successive filtering and down-sampling: $c_{i-1}[\boldsymbol{k}]=\left(h * c_{i}\right)[\mathbf{D} \boldsymbol{k}]$. The (symmetric) lowpass filter $h$ is specified by the two-scale relation for the polyharmonic B-spline

$$
\beta_{\gamma}\left(\mathbf{D}^{-1} \boldsymbol{x}\right)=|\operatorname{det}(\mathbf{D})|^{\frac{1}{2}} \sum_{\boldsymbol{k} \in \mathbb{Z}^{2}} h[\boldsymbol{k}] \beta_{\gamma}(\boldsymbol{x}-\boldsymbol{k})
$$

and its frequency response is found by inserting the formula for $\hat{\beta}_{\gamma}(\boldsymbol{\omega})$ provided by (34)

$$
\begin{aligned}
H\left(e^{j \boldsymbol{\omega}}\right) & =|\operatorname{det} \mathbf{D}|^{\frac{1}{2}} \frac{\hat{\beta}_{\gamma}\left(\mathbf{D}^{T} \boldsymbol{\omega}\right)}{\hat{\beta}_{\gamma}(\boldsymbol{\omega})} \\
& =|\operatorname{det} \mathbf{D}|^{\frac{1}{2}-\gamma}\left(\frac{V\left(e^{j \mathbf{D}^{T} \boldsymbol{\omega}}\right)}{V\left(e^{j \boldsymbol{\omega}}\right)}\right)^{\gamma / 2}
\end{aligned}
$$

where $V\left(e^{j \boldsymbol{\omega}}\right)$ is the discrete Laplacian filter defined by (35). Finally, the wavelet coefficients are obtained by digital filtering of the $c_{i}$ 's (without down-sampling)

$$
w_{i}[\boldsymbol{k}]=\left\langle f, \psi_{i, \boldsymbol{k}}\right\rangle=\left(c_{i} * g\right)[\boldsymbol{k}] .
$$

The wavelet analysis filter is determined from the following B-spline representation of the isotropic wavelet

$$
\begin{aligned}
& \psi_{\text {iso }}\left(\mathbf{D}^{-1} \boldsymbol{x}\right) \\
& \quad=|\operatorname{det}(\mathbf{D})|^{\frac{1}{2}} \sum_{\boldsymbol{k} \in \mathbb{Z}^{2}} g[\boldsymbol{k}] \beta_{\gamma}(\boldsymbol{x}-k)=(-\Delta)^{\frac{\gamma}{2}} \beta_{2 \gamma}(\boldsymbol{x}) .
\end{aligned}
$$

Its frequency response is obtained by taking the Fourier transform of this equation, which yields

$$
\begin{aligned}
G\left(e^{j \boldsymbol{\omega}}\right) & =|\operatorname{det} \mathbf{D}|^{-\frac{1}{2}} \frac{\|\boldsymbol{\omega}\|^{\gamma} \hat{\beta} 2 \gamma(\boldsymbol{\omega})}{\hat{\beta}_{\gamma}(\boldsymbol{\omega})} \\
& =|\operatorname{det} \mathbf{D}|^{-\frac{1}{2}} V\left(e^{j \boldsymbol{\omega}}\right)^{\gamma / 2}
\end{aligned}
$$

The decomposition algorithm for the Riesz counterpart of the transform is completely analogous and the process is perfectly reversible in both cases. Since the analysis and synthesis filters are nonseparable and specified by their frequency responses, we have chosen to implement all filtering and re-sampling operations in the Fourier domain assuming periodic boundary conditions. Our wavelet code uses FFTs; it is generic and quite efficient computationally. Matlab and JAVA (e.g., ImageJ plugin) versions of these transforms are available on the web at http:// bigwww.epfl.ch/demo/monogenic/.

\section{Wavelets Versus Scale Space}

Wavelets and scale space methods are distinct approaches to multiscale signal analysis; they both have their proponents and specialized conferences devoted to them. Interestingly, we can make a connection between the present approach and the $\alpha$-scale space of Duits, Felsberg and collaborators because both representations are tightly coupled to the fractional Laplacian operator [15], [44]. Specifically, these authors perform a monogenic analysis in a scale space that is governed by the fractional diffusion equation

$$
\frac{\partial f(\boldsymbol{x}, s)}{\partial s}=-(-\Delta)^{\alpha} f(\boldsymbol{x} ; s)
$$

with $\alpha \in(0,1]$ and the initial condition $f(x ; 0)=f(x)$. The corresponding monogenic scale-space flow is obtained by convolving the initial signal $f$ and its Riesz transform with a series of $\alpha$-stable smoothing kernels $K_{s}^{\alpha}(x)$ whose width increases with the scale $s$. For $\alpha=1 / 2$, the smoothing function is the Poisson kernel $K_{s}^{1 / 2}(\boldsymbol{x})=(s /(2 \pi)) /\left(s^{2}+\|\boldsymbol{x}\|^{2}\right)^{(d+1) / 2}$, while it is a Gaussian with a variance proportional to $s$ for $\alpha=1$. Therefore, apart from the Laplacian factor in (37), the scale-space version of the monogenic signal is qualitatively similar to the monogenic wavelet transform developed in this paper. However, there are four fundamental differences, which are at the very heart of wavelet theory:

a) the proposed wavelet transform uses subsampling and is a much less redundant representation;

b) it is a fully reversible representation;

c) it is a Hilbert-space method that involves a series of embedded approximation spaces;

d) the smoothing kernels at different scales are dyadic dilations of a single reference template (B-spline).

As a result, the proposed wavelet scheme is much more efficient computationally and storage-wise. The Poisson scale space, on the other hand, offers a more progressive scale progression; it is perfectly isotropic and predominantly lowpass [15]. 


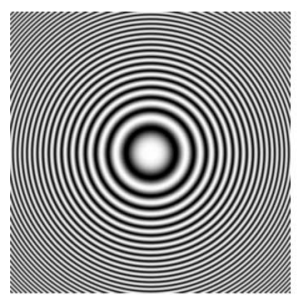

Synthetic zoneplate
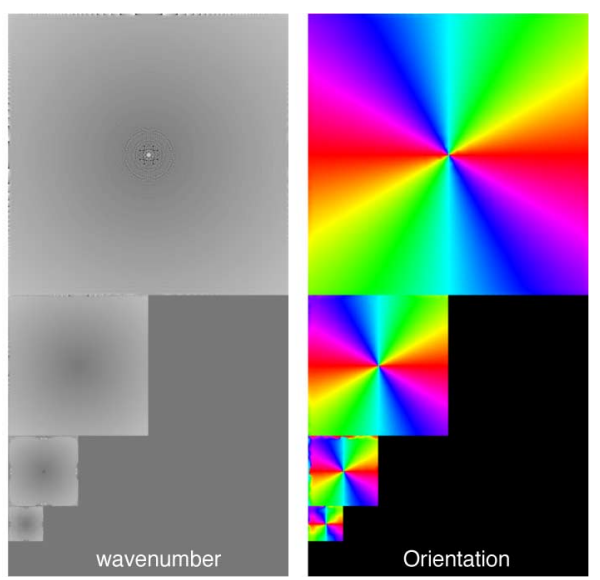

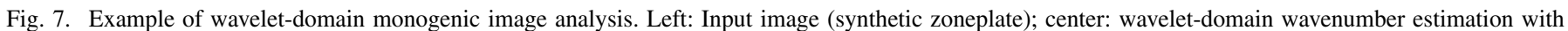
calibrated frequency scale. Right: Tensor-based orientation (Hue channel).

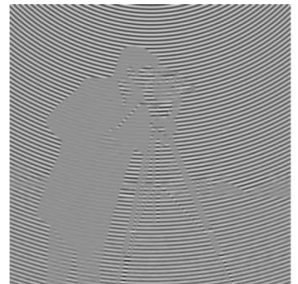

(a)
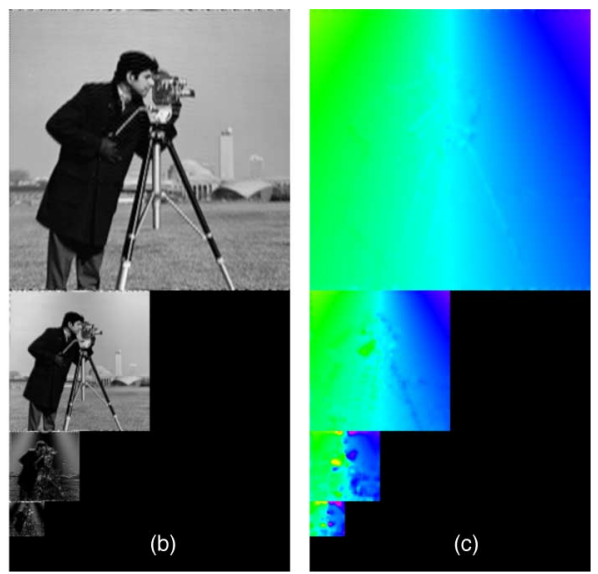

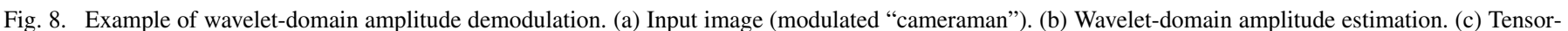
based orientation (Hue channel).

\section{Experimental Results}

All results presented here were obtained by performing a three-component monogenic Riesz-Laplace transform with $\gamma=2$ using the "improved" Mexican-hat-like analysis wavelet (Laplacian of a B-spline) described in Section V-A. We systematically used a tensor-based estimation of the orientation with a fixed Gaussian neighborhood window $(\sigma=2)$. The displayed orientation is wrapped within the interval $[0, \pi]$ and encoded in the hue channel; this color coding is exemplified with the circular pattern in Fig. 7, which contains a full 360-degree range of orientations.

The example in Fig. 8 illustrates the ability of the monogenic wavelet transform to recover amplitude-modulated signals when the frequency/phase of the carrier is unknown and/or slowly-varying. The test signal was generated by multiplying the standard "cameraman" image with a circular wave propagating with constant frequency. We observe that the AM-encoded information is retrieved in the amplitude of the transform and that the original image is identifiable down to the third level of resolution. A remarkable aspect is the robustness of the scheme with respect to scale, especially if one considers that the wavelet channels are bandpass filtered. Likewise, the direc- tion of the carrier is adequately captured by the wavelet orientation. We also notice some ghosting effect in the orientation map, which corresponds to the edges of the cameraman. The best separation is achieved at the second level of the pyramid, which yields the maximum amplitude response.

The synthetic chirp-like zoneplate image (cf. Fig. 7) was generated specifically to test the validity of the wavelet-domain local frequency estimation algorithm provided by (32). The orientation is properly extracted at all levels, as is obvious from Fig. 7(c). More importantly, we verified that the wavenumber was correctly estimated at all scales and locations: it is increasing linearly from the center outwards. Note that the finer-scale frequency estimation fails in the center of the image which is understandable since it is the region where the attenuation effect of highpass wavelet filtering is the strongest. In general, one should expect the reliability of a given wavenumber estimate to improve as its value gets closer to the center frequency of the corresponding wavelet band.

Fig. 9 provides another striking example of wavelet-domain AM/FM analysis. The input picture Barbara contains several textured objects (jeans, table cloth, etc.) which are segmented out by the multiscale coherency analysis. Note how the periodic structures give rise to near-constant white patches in the 


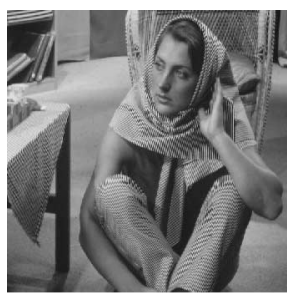

(a)
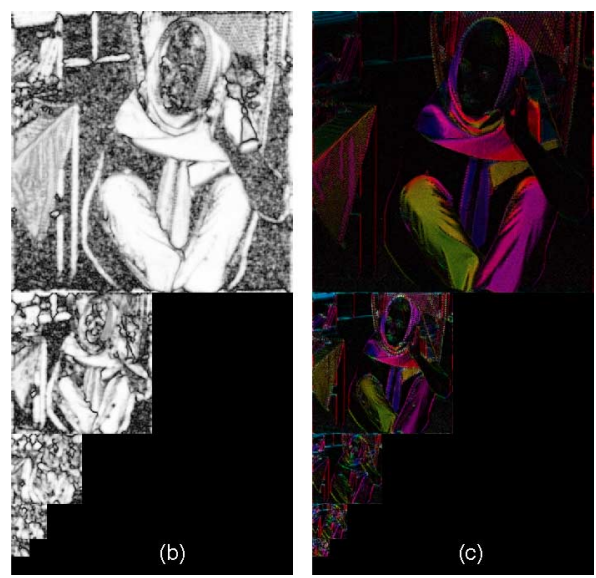

Fig. 9. Example of wavelet-domain coherence analysis. (a) $512 \times 512$ Barbara; (b) wavelet-domain coherency map; (c) composite HSB display of results (Hue: orientation, Saturation: coherency, Brightness: amplitude).

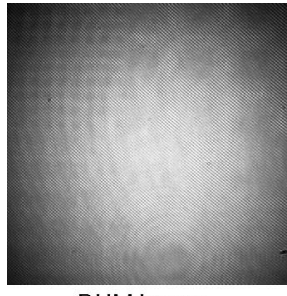

DHM image
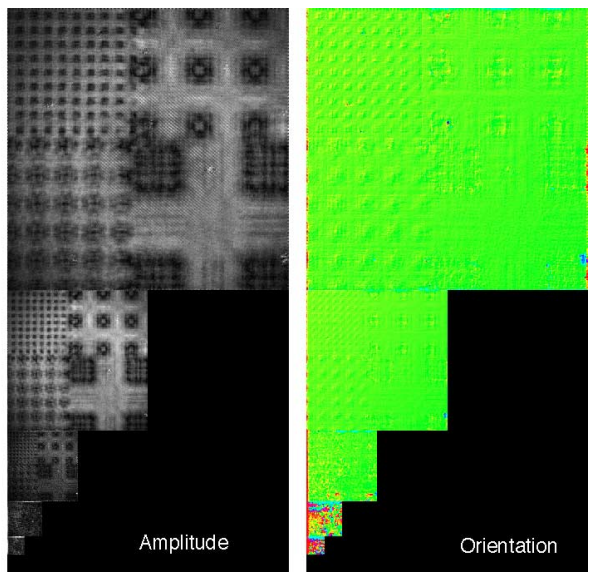

Fig. 10. Example of wavelet-domain amplitude demodulation. Left: Experimental digital holography micrograph; center: wavelet-domain amplitude estimation. Right: Tensor-based orientation (Hue channel).

coherency map and to nicely saturated colored regions in the composite orientation display. The crisper aspect of the latter is due to its brightness being controlled by the wavelet amplitude; this is a more local feature than the coherency which is defined over a local neighborhood. The primary contours of the picture are also properly detected in the multiscale maps.

The next example illustrates the applicability of the technique to the processing of real-world interferometric data. The image on the left of Fig. 10 was acquired using a digital holography microscope. It is a hologram that results from the interference between the wave reflected by the object to be imaged (a patterned nano-structure) and a reference plane wave (or carrier) [45]. The light intensity of the object can be reconstructed by using an appropriate demodulation and filtering algorithm [46]. Fig. 10 illustrates the ability of the monogenic wavelet transform to perform such a blind multiresolution reconstruction via its wavelet amplitude. The orientation map indicates that the carrier is a diagonally-oriented plane wave. We can also estimate its frequency from the wavenumber map (data not shown) which is essentially constant within the first three bands. Note that the transform also separates out some artifactual lower-frequency interferences that are superimposed onto the input signal and partly visible as circular patterns. In the present case, these components get transfered into the two lower resolution levels of the pyramid; they are probably due to some dust particle intersecting the optical path of the laser beam.

The final illustration is the multiresolution analysis of a fingerprint image which contains oriented ridge-like structures (cf. Fig. 11). At finer scale, the wavelet modulus acts as a contour detector, while it eventually fills in the whole finger print area as the scale gets coarser (level 3). The multiresolution orientation map nicely captures the directionality of the underlying pattern; it also gets smoother and less noisy with increasing scale. The third level estimate is probably the one that looks the most satisfactory from a visual point of view. For more advanced feature extraction, we refer to the work of Larkin et al. which describes a related phase-based framework for fingerprint analysis; it includes specific detectors for branch cuts, loops and delta structures [47].

\section{CONCLUSION}

We have set the mathematical foundations of a monogenic wavelet transform that gives access to the local orientation, amplitude and phase information of a 2-D signal within a proper multiresolution setting. The transform is build around the monogenic extension of a polyharmonic spline wavelet 


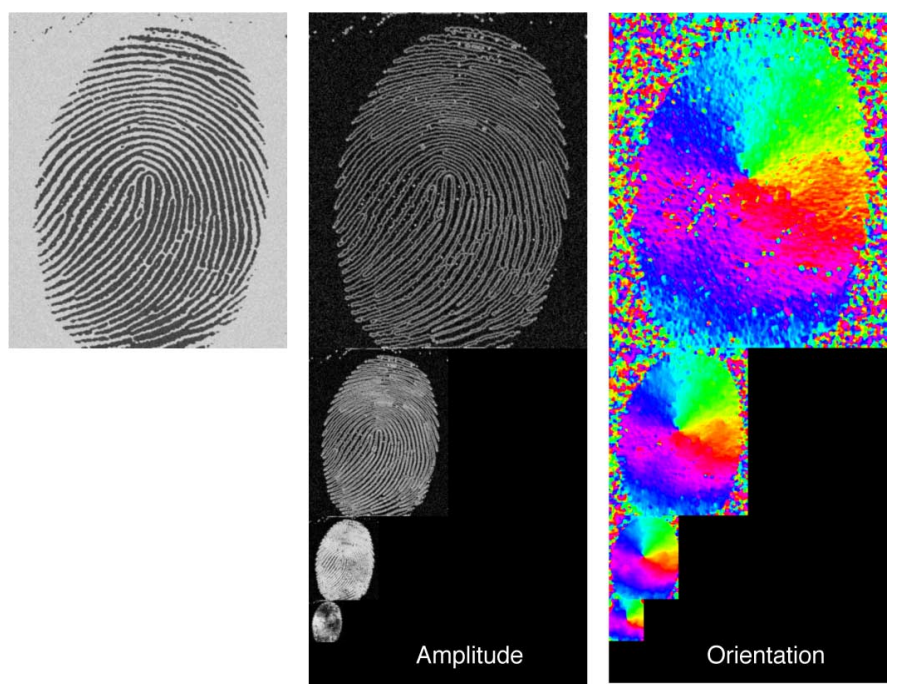

Fig. 11. Example of wavelet-domain monogenic image analysis. Left: Original fingerprint image; center: wavelet-domain amplitude estimation. Right: Tensorbased orientation (Hue channel).

basis of $L_{2}\left(\mathbb{R}^{2}\right)$. The decomposition is reasonably fast, moderately redundant (a factor 3 or 4 , depending on the sampling configuration), and fully reversible.

An important property is that the complex Riesz branch of the transform, which is akin to a multiscale gradient, is steerable. We have shown that this decomposition can yield a new set of tensor-based features (orientation, Hilbert-transform energy, and coherency) that offer interesting perspectives for local orientation, texture and structure analysis; this extraction process is robust and essentially rotation-invariant. The latter property is not fulfilled by the great majority of features obtained by conventional separable wavelet processing.

The distinguishing aspect of the present decomposition, when we compare it to the other currently-available complex or quaternion wavelet transforms, is the presence of the Laplacian branch of the transform, which yields the primary component in the monogenic signal formalism. It essentially acts like an isotropic filterbank which decomposes the signal into a series nondirectional wavelet subbands. The complementary role of the Riesz wavelets is to estimate the predominant local orientation within the given band and to compute the corresponding directional Hilbert transform through an appropriate steering mechanism. The monogenic feature extraction process ultimately gives access to the modulation parameters of the signal: amplitude, phase and instantaneous frequency, which are difficult to extract otherwise. Potential applications include various type of coherent image analyses-in particular, the processing of interferograms and fringe patterns-as well as image enhancement and denoising since the transform is reversible. More generally, one may build upon the important body of work on the monogenic signal and take advantage of the Riesz-Laplace transform for transposing some of these methods to the wavelet domain, which may result in novel algorithms.

\section{APPENDIX I}

\section{DiRECTIONAL HILBERT ANALYSIS}

The monogenic wavelet components are denoted by $\left(w, r_{1}, r_{2}\right)$. Our goal is to determine the amplitude $(A)$, the phase $(\xi)$ and the instantaneous frequency $(\nu)$ in the direction specified by $\boldsymbol{u}=(\cos \theta, \sin \theta)$ where $\theta$ is our local orientation estimate-not necessarily equal to $\theta_{0}=\arctan \left(r_{2} / r_{1}\right)$. To that end, we first define

$$
q=r_{1} \cos \theta+r_{2} \sin \theta
$$

which is the Hilbert transform of $w$ in the direction $\theta$. Next, we introduce the complex signal $z=w+j q$, which is the directional counterpart of the analytic signal in 1-D. The amplitude and phase information is encoded in the polar representation $z=A e^{j \xi}$, which yields

$$
\begin{aligned}
A & =\sqrt{w^{2}+q^{2}} \\
\xi & =\arctan \left(\frac{q}{w}\right) .
\end{aligned}
$$

The instantaneous frequency $\nu$ is obtained by taking the derivative of $\xi$ along the direction of analysis: $\nu=\mathrm{D}_{\boldsymbol{u}} \xi$. To get a more practical formula, we first evaluate the phase gradient using the property that $\xi=\operatorname{Im}(\log z)$

$$
\nabla \xi=\operatorname{Im}\left(\frac{\nabla z}{z}\right)=\frac{-q \nabla w}{A^{2}}+\frac{w \nabla q}{A^{2}}
$$

with the differential notation $\nabla w=(\partial w / \partial x, \partial w / \partial y)=$ $\left(w_{x}, w_{y}\right)$. We obtain the directional derivative by taking the inner product with $\boldsymbol{u}$, which gives

$$
\mathrm{D}_{\boldsymbol{u}} \xi=\langle\nabla \xi, \boldsymbol{u}\rangle=\frac{-q\left(w_{x} \cos \theta+w_{y} \cos \theta\right)}{A^{2}}+\frac{w\left(\boldsymbol{u}^{T} \mathbf{H} \boldsymbol{u}\right)}{A^{2}}
$$

where $\mathbf{H}=\left(\begin{array}{ll}r_{1 x} & r_{1 y} \\ r_{2 x} & r_{2 y}\end{array}\right)$ is a symmetric matrix (analogous to the Hessian) due to the properties of the Riesz transform. If we now assume that the underlying signal is locally coherent (in the sense that it has a 1-D structure along the direction $\boldsymbol{u}$ ), then $\mathbf{H}$ is of rank 1 and $\boldsymbol{u}^{T} \mathbf{H} \boldsymbol{u}=\lambda_{\max }=\operatorname{trace}(\mathbf{H})=r_{1 x}+r_{2 y}$. This leads to the simplified formula

$$
\nu=\mathrm{D}_{\boldsymbol{u}} \xi=\frac{-q\left(w_{x} \cos \theta+w_{y} \sin \theta\right)}{A^{2}}+\frac{w\left(r_{1 x}+r_{2 y}\right)}{A^{2}} .
$$


Thus, the critical ingredient for this determination is to provide a numerical procedure for computing the derived quantities $w_{x}$, $w_{y}$ and $-\left(r_{1 x}+r_{2 y}\right)$, which can be done in an efficient way using two reduced-order wavelet transforms, as specified in the text.

\section{ACKNOWLEDGMENT}

The authors would like to thank K. Balac (KB) for her implementation of the Laplace/Riesz transform as a JAVA Plugin for ImageJ. They would also like to thank F. Aguet for providing the original test image (Fig. 1) and Prof. C. Depeursinge for giving access to his digital holography microscopy data.

\section{REFERENCES}

[1] D. Gabor, "Theory of communication," J. Inst. Elect. Eng., vol. 93, no. III, pp. 429-457, 1946.

[2] S. L. Hahn, Hilbert Transforms in Signal Processing. Boston, MA: Artech House, 1996.

[3] L. Moura and P. Monteiro, "Design method for FIR-based Hilbert transform filters suitable for broadband AM-SSB," Electron. Lett., vol. 38, no. 12, pp. 605-606, Jun. 6, 2002.

[4] N. Kingsbury, "Image processing with complex wavelets," Philos. Trans. Royal Soc. London A, Math. Phys. Eng. Sci., vol. 357, no. 1760, pp. 2543-2560, 1999.

[5] N. G. Kingsbury, "Complex wavelets for shift-invariant analysis and filtering of signals," J. Appl. Comput. Harmon. Anal., vol. 10, no. 3, pp. 234-253, May 2001.

[6] I. Selesnick, "Hilbert transform pairs of wavelet bases," IEEE Signal Process. Lett., vol. 8, no. 6, pp. 170-173, Jun. 2001.

[7] I. Selesnick, "The design of approximate Hilbert transform pairs of wavelet bases," IEEE Trans. Signal Process., vol. 50, no. 5, pp. 1144-1152, May 2002.

[8] K. Chaudhury and M. Unser, "Construction of Hilbert transform pairs of wavelet bases and Gabor-like transforms," IEEE Trans. Signal Processing, vol. 57, no. 9, pp. 3411-3425, Sep. 2009.

[9] S. Hahn, "Multidimensional complex signals with single-orthant spectra," Proc. IEEE, vol. 80, no. 8, pp. 1287-1300, Aug. 1992.

[10] T. Bülow and G. Sommer, "Hypercomplex signals-A novel extension of the analytic signal to the multidimensional case," IEEE Trans. Signal Process., vol. 49, no. 11, pp. 2844-2852, Nov. 2001.

[11] M. Felsberg and G. Sommer, "The monogenic signal," IEEE Trans. Signal Process., vol. 49, no. 12, pp. 3136-3144, Dec., 2001.

[12] I. W. Selesnick, R. G. Baraniuk, and N. C. Kingsbury, "The dual-tree complex wavelet transform," IEEE Sig. Process. Mag., vol. 22, no. 6 , pp. 123-151, 2005.

[13] W. L. Chan, H. Choi, and R. G. Baraniuk, "Coherent multiscale image processing using dual-tree quaternion wavelets," IEEE Trans. Image Process., vol. 17, no. 7, pp. 1069-1082, Jul. 2008.

[14] E. Stein and G. Weiss, Introduction to Fourier Analysis on Euclidean Spaces. Princeton, NJ: Princeton Univ. Press, 1971.

[15] M. Felsberg and G. Sommer, "The monogenic scale-space: A unifying approach to phase-based image processing in scale-space," J. Math. Imag. Vis., vol. 21, no. 1, pp. 5-26, 2004.

[16] D. Zang and G. Sommer, "Signal modeling for two-dimensional image structures," J. Vis. Commun. Image Represent., vol. 18, no. 1, pp. 81-99, 2007.

[17] K. Takaya, "Feature point correspondence of stereo images by monogenic phase," in Proc. IEEE Pacific Rim Conf. Communications, Computers and Signal Processing PacRim, 2007, pp. 272-275.

[18] M. Mellor and M. Brady, "Phase mutual information as a similarity measure for registration," Med. Image Anal., vol. 9, no. 4, pp. 330-343, Apr. 2005.

[19] R. Ali, M. Gooding, M. Christlieb, and M. Brady, "Advanced phasebased segmentation of multiple cells from brightfield microscopy images," in Proc. IEEE Int. Symp. Biomedical Imaging, Paris, France, May $14-17,2008$, pp. 181-184.
[20] K. G. Larkin, D. J. Bone, and M. A. Oldfield, "Natural demodulation of two-dimensional fringe patterns. I. General background of the spiral phase quadrature transform," J. Opt. Soc. Amer. A, vol. 18, no. 8, pp. $1862-1870,2001$.

[21] K. G. Larkin, "Natural demodulation of two-dimensional fringe patterns. II. Stationary phase analysis of the spiral phase quadrature transform," J. Opt. Soc. Amer. A, vol. 18, no. 8, pp. 1871-1881, 2001.

[22] J. A. Guerrero, J. L. Marroquin, M. Rivera, and J. A. Quiroga, "Adaptive monogenic filtering and normalization of ESPI fringe patterns," Opt. Lett., vol. 30, no. 22, pp. 3018-3020, 2005.

[23] G. Metikas and S. C. Olhede, "Multiple multidimensional Morse wavelets," IEEE Trans. Signal Process., vol. 55, no. 3, pp. 921-936, Mar. 2007.

[24] D. Boukerroui, J. A. Noble, and M. Brady, "On the choice of band-pass quadrature filters," J. Math. Imag. Vis., vol. 21, no. 1, pp. 53-80, 2004

[25] S. Held, M. Storah, P. Massopust, and B. Forster, "Steerable wavelet frames based on the Riesz transform," IEEE Trans. Image Process., to be published.

[26] W. T. Freeman and E. H. Adelson, "The design and use of steerable filters," IEEE Trans. Pattern Anal. Mach. Intell., vol. 13, no. 9, pp. 891-906, Sep. 1991.

[27] B. Jähne, Digital Image Processing. New York: Springer, 2005.

[28] I. Gelfand and G. Shilov, Generalized Functions. New York: Academic, 1964, vol. 1.

[29] F. Natterer, The Mathematics of Computed Tomography. New York: Wiley, 1984.

[30] A. C. Kak and M. Slaney, Principles of Computerized Tomographic Imaging. New York: IEEE, 1987

[31] L. Wietzke and G. Sommer, The 2D Analytic Signal Christian-Albrechts-Universität zu Kiel, Tech. Rep. 0802, 2008.

[32] W. R. Madych and S. A. Nelson, "Polyharmonic cardinal splines," $J$. Approx. Theory, vol. 60, no. 2, pp. 141-156, 1990

[33] A. Aldroubi and M. Unser, "Sampling procedures in function spaces and asymptotic equivalence with Shannons sampling theory," Numer. Funct. Anal. Optim., vol. 15, no. 1-2, pp. 1-21, 1994.

[34] D. Van De Ville, T. Blu, and M. Unser, "Isotropic polyharmonic B-splines: Scaling functions and wavelets," IEEE Trans. Image Process., vol. 14, no. 11, pp. 1798-1813, Nov. 2005.

[35] B. Bacchelli, M. Bozzini, C. Rabut, and M. L. Varas, "Decomposition and reconstruction of multidimensional signals using polyharmonic prewavelets," Appl. Comput. Harmon. Anal., vol. 18, no. 3, pp. 282-299, 2005

[36] M. D. Buhmann, Radial Basis Functions. Cambridge, U.K.: Cambridge Univ. Press, 2003

[37] C. Rabut, "Elementary m-harmonic cardinal B-splines," Numer. Alg., vol. 2, pp. 39-62, 1992.

[38] C. Micchelli, C. Rabut, and F. Utreras, "Using the refinement equation for the construction of pre-wavelets III: Elliptic splines," Numer. Alg., vol. 1, pp. 331-352, 1991

[39] B. Forster, T. Blu, D. Van De Ville, and M. Unser, "Shift-invarian spaces from rotation-covariant functions," Appl. Comput. Harmon. Anal., vol. 25, no. 2, pp. 240-265, 2008

[40] D. Van De Ville and M. Unser, "Complex wavelet bases, steerability, and the Marr-like pyramid," IEEE Trans. Image Process., vol. 17, no 11, pp. 2063-2080, Nov. 2008.

[41] M. Unser and T. Blu, "Wavelet theory demystified," IEEE Trans. Signal Process., vol. 51, no. 2, pp. 470-483, Feb. 2003.

[42] C. Harris and M. Stephens, "A combined corner and edge detector," in Proc. Alvey Vision Conf., 1988, pp. 147-152.

[43] M. Unser and D. Van De Ville, "The pairing of a wavelet basis with a mildly redundant analysis via subband regression," IEEE Trans. Image Process., vol. 17, no. 11, pp. 2040-2052, Nov. 2008.

[44] R. Duits, M. Felsberg, L. Florack, and B. Platel, " $\alpha$ scale spaces on a bounded domain," in Proc. Scale Space'03, ser. Lecture Notes in Computer Science. Heidelberg, Germany: Springer, 2003, vol. 2695, pp. 502-518.

[45] E. Cuche, F. Bevilacqua, and C. Depeursinge, "Digital holography for quantitative phase-contrast imaging," Opt. Lett., vol. 24, no. 5, pp 291-293, 1999.

[46] E. Cuche, P. Marquet, and C. Depeursinge, "Spatial filtering for zeroorder and twin-image elimination in digital off-axis holography," Appl. Opt., vol. 39, no. 23, pp. 4070-4075, 2000.

[47] K. G. Larkin and P. A. Fletcher, "A coherent framework for fingerprint analysis: Are fingerprints holograms?," Opt. Exp., vol. 15, no. 14, pp. 8667-8677, 2007. 


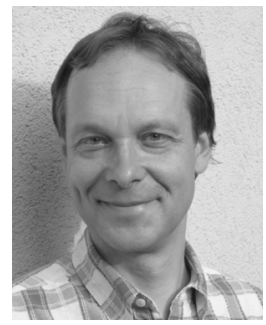

Michael Unser (M'89-SM'94-F'99) received the M.S. (summa cum laude) and Ph.D. degrees in electrical engineering in 1981 and 1984, respectively, from the École Polytechnique Fédérale de Lausanne (EPFL), Switzerland.

From 1985 to 1997, he was a Scientist with the National Institutes of Health, Bethesda, MD. He is now full Professor and Director of the Biomedical Imaging Group, EPFL. His main research area is biomedical image processing. He has a strong interest in sampling theories, multiresolution algorithms, wavelets, and the use of splines for image processing. He has published over 150 journal papers on those topics and is one of ISI's Highly Cited authors in Engineering (http://isihighlycited.com).

Dr. Unser has held the position of associate Editor-in-Chief (2003-2005) for the IEEE TRANSACTIONS ON MEDICAL IMAGING and has served as Associate Editor for the same journal (1999-2002; 2006-2007), the IEEE TRANSACTIONS ON IMAGE PROCESSING (1992-1995), and the IEEE SignAL PROCESSING LETTERS (1994-1998). He is currently member of the editorial boards of Foundations and Trends in Signal Processing, the SIAM Journal of Imaging Sciences, and Sampling Theory in Signal and Image Processing. He co-organized the first IEEE International Symposium on Biomedical Imaging (ISBI2002). He was the founding chair of the technical committee of the IEEE-SP Society on Bio Imaging and Signal Processing (BISP). He received the 1995 and 2003 Best Paper Awards and the 2000 Magazine Award from the IEEE Signal Processing Society.

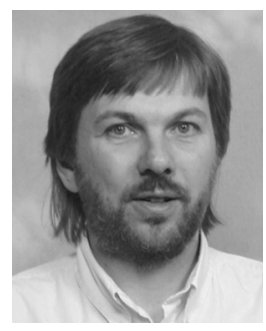

Daniel Sage received the M.S. and Ph.D. degrees in control and signal processing from the Institut $\mathrm{Na}$ tional Polytechnique de Grenoble (INPG), Grenoble, France, in 1986 and 1989, respectively.

From 1989 to 1998, he was a Consulting Engineer in the Industrial Vision Department, Attexor S.A. During his career, he has developed vision systems oriented to the quality control in the industrial sector. In 1998, he joined the Biomedical Imaging Group at the Ecole Polytechnique Fédérale de Lausanne (EPFL), Lausanne, Switzerland, as the Head of software development. He is in charge of both the coordination of software development and of setting down the computing infrastructure of the group. $\mathrm{He}$ is also involved in the development of bio-imaging software and methods for computer-assisted teaching.

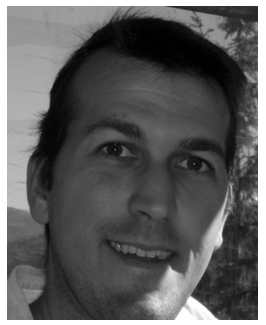

Dimitri Van De Ville (M'02) received the M.S. degree in engineering and computer sciences from Ghent University, Ghent, Belgium, in 1998, and the Ph.D. degree in 2002.

He received a grant as Research Assistant with the Fund for Scientific Research Flanders Belgium (FWO). In 2002, he joined Prof. M. Unser's Biomedical Imaging Group at the École Polytechnique Fédérale de Lausanne (EPFL), Lausanne, Switzerland. Since December 2005, he has been heading the Signal Processing Unit at the University Hospital of Geneva, Geneva, Switzerland, as part of the Centre d'Imagerie Biomédicale (CIBM). He is the recipient of an assistant professor fellowship from the Swiss National Science Foundation. His research interests include wavelets, statistical analysis, multidimensional splines, and applications in biomedical imaging, such as functional magnetic resonance imaging, spectroscopy, electro-encephalography, and microscopy.

Dr. Van De Ville serves as an Associate Editor for the IEEE TRANSACTIONS ON IMAGE PROCESSING (since February 2006) and was previously an Associate Editor for IEEE SIGNAL PROCESSING LETTERS (2004-2006). Since 2003, he has also been an Editor and Webmaster of The Wavelet Digest. He is co-chair of the Wavelets XII (2007) Wavelets XIII (2009) international conferences, together with V. Goyal and M. Papadakis. 